\title{
REAL ALGEBRAIC AND PSEUDOHOLOMORPHIC CURVES ON THE QUADRATIC CONE AND SMOOTHINGS OF SINGULARITY $X_{21}$
}

\author{
S. Yu. Orevkov and E. I. Shustin \\ Dedicated to Sergei Vladimirovich Vostokov, the first supervisor of the second author
}

\begin{abstract}
We complete a fiberwise isotopy classification of smooth real algebraic and pseudoholomorphic curves of degree 8 on the quadratic cone, which have a specially shaped oval crossing a given generating line of the cone in four real points. We link this classification with an isotopy classification of smoothing of real plane curve singularity which is the union of four smooth real local branches quadratically tangent to each other (the singularity $X_{21}$ ).
\end{abstract}

\section{INTRODUCTION}

Problem and results. Let $\mathcal{F}_{2}$ be the second Hirzebruch surface, i.e. the ruled surface possessing an exceptional (-2)-curve (which is the same as the quadratic cone with the singular point blown up) and equipped with the standard real structure. Denote the $(-2)$-curve by $E$, a section disjoint from $E$ by $H$, and the ruling by $\pi: \mathcal{F}_{2} \rightarrow E$. We fix some real fiber $F_{0}$ (throughout the paper, fibers of $\pi$ are called just fibers).

In this paper we complete the classification of the pairs $\left(C, C \cap F_{0}\right)$ in $\left(\mathcal{F}_{2}, F_{0}\right)$, where $C \in|4 H|$ is a smooth real curve having an oval, which intersects $F_{0}$ in four distinct real points as shown in Figure 1, and the equivalence is defined up to an almost fiberwise isotopy of $\left(\mathbb{R} C, \mathbb{R} C \cap F_{0}\right)$ in $\left(\mathbb{R} \mathcal{F}_{2}, \mathbb{R} F_{0}\right)$, i.e., a $C^{\infty}$-isotopy that keeps $\mathbb{R} E$ fixed and satisfies the condition that each real fiber intersects any curve in the isotopy in at most 4 points counting multiplicities. In particular, an almost fiberwise isotopy preserves the relative position of free ovals with respect to the fibers (here and below the ovals of $C$ disjoint from $F_{0}$ are called free). In a

1991 Mathematics Subject Classification. Primary 14P25, 57M25; Secondary 14H20, 53D99.

The first author has been supported by RSF grant, project 14-21-00053 dated 11.08.14.

The second author has been supported by the German-Israeli Foundation, grant no. 1174197.6/2011, and by the Hermann-Minkowski-Minerva Center for Geometry at the Tel Aviv University. The main part of this work has been performed during the second author's visit to the Centre Interfacultaire Bernoulli at the École Polytechnique Fedérale de Lausanne in March-May 2015 and to the Max-Planck Institut für Mathematik, Bonn, in August-September 2015. The second author is very grateful to CIB-EPFL and MPI for hospitality and excellent working conditions.

Special thanks are due to the referee, who pointed out several mistakes in the preliminary version of the paper. 
similar way one can classify smooth real pseudoholomorphic curves homologous to $4 H$. It was pointed out in [25] that these classifications differ, i.e. there are real pseudoholomorphic curves which are not almost fiberwise isotopic to any real algebraic curve in the considered class. Both classifications are presented in Theorem 1 below.

An important remark is that by a real pseudo-holomorphic curve on $\mathcal{F}_{2}$ we always mean a conj-invariant $J$-holomorphic curve where $J$ is a tame conj-anti-invariant almost complex structure on $\mathcal{F}_{2}$ such that the $(-2)$-section is J-holomorphic. Other settings where some real pseudoholomorphic curves are algebraically unrealizable are discussed in $[4,8,20,23,33]$.

We also prove (see Theorem 5 in Section 6) that the almost fiberwise algebraic classification is equivalent to the almost fiberwise classification of smoothings of the real plane curve singularity $X_{21}$ (i.e., four real smooth local branches quadratically tangent to each other). Notice that smoothings of $X_{21}$ play an important role in the classification of smooth real plane curves of degree 8 and $9[16,31,32]$ - a part of Hilbert's 16th problem.

Classification. Suppose that $C$ is a real smooth algebraic or pseudoholomorphic curve on $\mathcal{F}_{2}$, homologous to $4 H$, and $\mathbb{R} C$ has a connected component intersecting the fiber $F_{0}$ in four points as shown in Figure 1 , where $\mathbb{R} F_{0}$ is represented by the vertical sides of the rectangles and $E$ is represented by the horizontal sides. We say that such a connected component is of type $\mathcal{A}$. By [25; Corollary 2.3] the other ovals of $C$ are empty, and their almost fiberwise arrangement (up to rotation by $180^{\circ}$ ) is either $\mathcal{A}^{\prime}(a, b, c)$ or $\mathcal{A}^{\prime \prime}(a, b, c)$, see Figure 1 where the $a, b$, and $c$ are non-negative integers which represent the corresponding amounts of empty ovals.
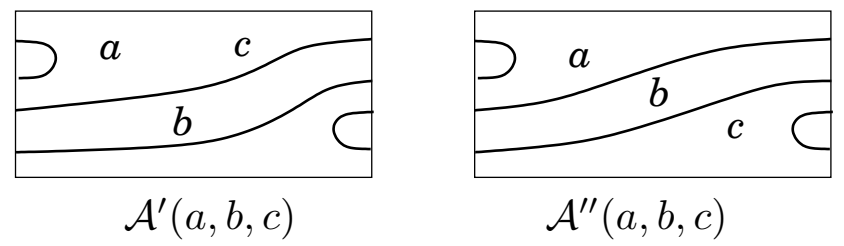

Figure 1

Theorem 1. (a) Let $C \in|4 H|$ be a smooth algebraic curve with an oval of type $\mathcal{A}$. Then the almost fiberwise arrangement of ovals of $C$ is either one of

$$
\begin{array}{ll}
\mathcal{A}^{\prime}(0,1,8), \mathcal{A}^{\prime}(6,1,2), \mathcal{A}^{\prime}(0,5,4), \mathcal{A}^{\prime}(2,5,2), & M \text {-curves } \\
\mathcal{A}^{\prime \prime}(7,0,1), \mathcal{A}^{\prime \prime}(3,4,1), & (M-1) \text {-curves } \\
\mathcal{A}^{\prime}(0,2,5), \mathcal{A}^{\prime}(3,2,2), \mathcal{A}^{\prime}(4,2,1), \mathcal{A}^{\prime}(3,3,1), \mathcal{A}^{\prime}(0,6,1), & (M-2) \text {-curves } \\
\mathcal{A}^{\prime \prime}(4,3,0), \mathcal{A}^{\prime \prime}(0,7,0), \mathcal{A}^{\prime \prime}(5-b, b, 2), b=0,1,2,3, & (M-2) \text {-curves } \\
\mathcal{A}^{\prime}(1,1,3) & (M-4) \text {-curve }
\end{array}
$$

or it is obtained from them by removing some free ovals. All these arrangement are realizable by algebraic curves.

(b) Let $C$ be a smooth pseudoholomorphic curve homologous to $4 H$ and having an oval of type $\mathcal{A}$. Then the fiberwise arrangement of ovals of $C$ is either one of 
Part (a) or one of:

$$
\begin{array}{ll}
\mathcal{A}^{\prime \prime}(4,1,4), & M \text {-curves } \\
\mathcal{A}^{\prime \prime}(4,0,4)^{*}, \mathcal{A}^{\prime \prime}(5,0,3), \mathcal{A}^{\prime \prime}(6,0,2), \mathcal{A}^{\prime \prime}(4,1,3)^{*}, & (M-1) \text {-curves } \\
\mathcal{A}^{\prime \prime}(4,0,3)^{*}, \mathcal{A}^{\prime \prime}(3,1,3)^{*} & (M-2) \text {-curves } \\
\mathcal{A}^{\prime \prime}(3,0,3)^{*} & (M-3) \text {-curve }
\end{array}
$$

(the arrangements marked by the asterisk are obtained from the others by removing free ovals). All these arrangement are realizable by pseudoholomorphic curves.

Let $\mathcal{A}(a, b, c)$ be the isotopy type of $\mathcal{A}^{\prime \prime}(a, b, c)$ where we consider isotopy equivalence in $\mathbb{R} \mathcal{F}_{2}$ relative to $\mathbb{R} F_{0} \cup \mathbb{R} E$, which does not respect fibers. So, $\mathcal{A}(a, b, c)=$ $\mathcal{A}^{\prime \prime}(a, b, c)$ for $c \neq 0$, and $\mathcal{A}(a, b, 0)=\bigcup_{a_{1}+c_{1}=a} \mathcal{A}^{\prime}\left(a_{1}, b, c_{1}\right)$.

Corollary 2. An isotopy type $\mathcal{A}(a, b, c)$ is realizable by an algebraic (resp. pseudoholomorphic) curve if and only if $a \leq a_{1}, b \leq b_{1}, c \leq c_{1}$ where either $\left(a_{1}, b_{1}, c_{1}\right)$ or $\left(c_{1}, b_{1}, a_{1}\right)$ belongs to

$$
\begin{gathered}
S=\{(8,1,0),(4,5,0),(7,0,1),(3,4,1),(5,0,2),(4,1,2), \\
(3,2,2),(5,2,0),(2,3,2),(1,6,0),(0,7,0)\}
\end{gathered}
$$

(resp. to $S \cup\{(4,1,4),(5,0,3),(6,0,2)\})$.

Corollary 3. If $a+b+c \leq 5$, then $\mathcal{A}^{\prime}(a, b, c)$ and $\mathcal{A}^{\prime \prime}(a, b, c)$ are algebraically realizable.

Corollary 4. The almost fiberwise arrangements listed in Theorem 1(b) are unrealizable by a smooth real analytic deformation of singularity $X_{21}$ (four quadratically tangent smooth local branches).

Proof. Combine Theorem 1(a) and Theorem 5 (see Section 6).

We would like to mention that the spoken classification has been addressed earlier: in [25; Theorem 1], we have completed the classification of types $\mathcal{A}(a, b, c)$ with $a+b+c=9$ ( $M$-types). In [25], we also advanced in the classification of $(M-1)$-types leaving the types $\mathcal{A}^{\prime \prime}(6,0,2)$ and $\mathcal{A}^{\prime \prime}(3,1,4)$ undecided. Later in $[26$; Theorem 1.2] we prohibited algebraic curves of type $\mathcal{A}^{\prime \prime}(3,1,4)$.

\section{Constructions}

Construction of pseudoholomorphic curves. The $M$ - and $(M-1)$-curves in Theorem 1(b) are constructed in $[25 ; \S 4.2]$.

Construction of algebraic curves. The four $M$-arrangements in Theorem 1(a) are constructed in $[32 ; \S 4.7]$ and $[25 ; \S 4.1]$. The construction from $[25 ; \S 4.1]$ with other ways of gluing the charts of cubic polynomials provides the other arrangements of the series $\mathcal{A}^{\prime}$ listed in Theorem 1 (a) except $\mathcal{A}^{\prime}(3,3,1)$.

The two $(M-1)$-arrangements in Theorem 1 (a) are constructed in $[25 ; \S 2.4$, Corollary 2.12] (earlier they were constructed by the second author [28] and by Korchagin [15]).

The remaining arrangements in Theorem $1(\mathrm{a})$, i. e., $\mathcal{A}^{\prime}(3,3,1)$ and the six $(M-$ 2 )-arrangements of the series $\mathcal{A}^{\prime \prime}$, are constructed in Propositions 1.2, 1.5, and 1.8 below. One can easily check that all the constructions admit the erasing of any collection of empty ovals. 
Lemma 1.1. There exists a real algebraic curve in the linear system $|4 H|$ on $\mathcal{F}_{2}$ with singular points of types $A_{4}$ and $D_{13}$ arranged as in Figure 2(a) up to isotopy.

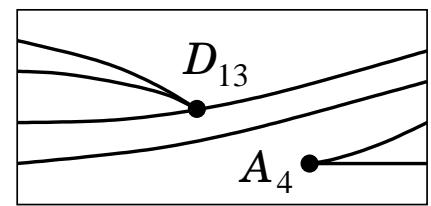

(a)

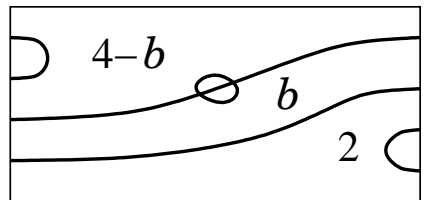

(b)

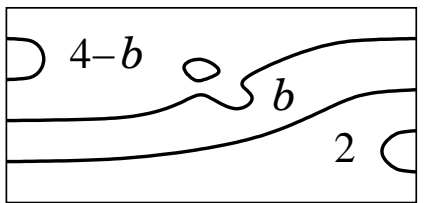

(c)

FiguRE 2

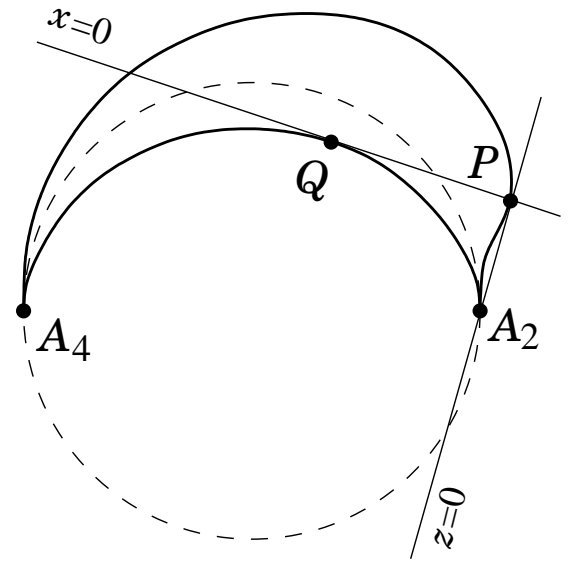

(a)

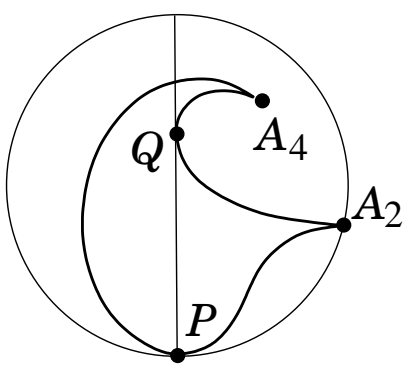

(b)

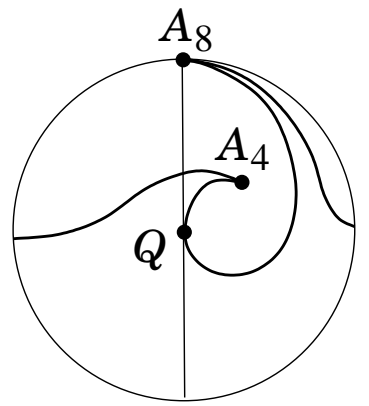

(c)

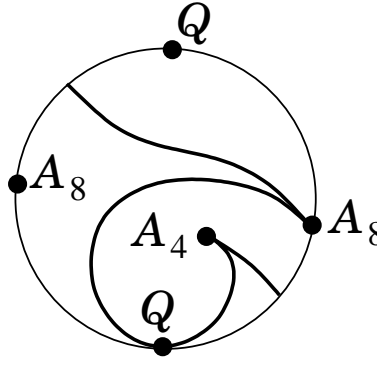

(d)

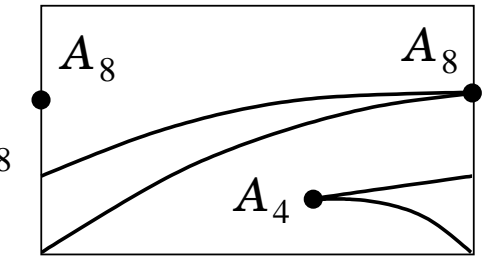

(e)

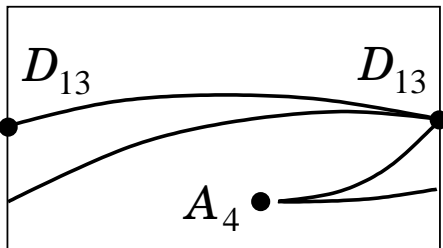

(f)

FiguRE 3

Proof. The construction is shown in Figure 3. We start with a quartic curve in $\mathbb{R} P^{2}$ with $A_{2}$ and $A_{4}$ singularities (it can be constructed, e. g., by a perturbation of a double conic). We choose coordinates $(x: y: z)$ as in Figure 3(a). In Figure 3(b) we show the same curve but $\mathbb{R} P^{2}$ is represented as a disk whose boundary points are identified. Figure 3(c) is obtained from Figure 3(b) by a birationl transformation which is written as $(X, Y) \mapsto\left(X, Y+a X^{2}\right)$ in the affine coordinates $X=x / z$, $Y=y / z$. Figure $3(\mathrm{~d})$ is the same as Figure $3(\mathrm{c})$ but $\mathbb{R} P^{2}$ is cut along another line. Figure 3(e) is obtained from it by blowing up the point $Q$ (the horizontal sides of the rectangle represent the $(-1)$-curve) and Figure $3(\mathrm{f})$ is obtained from Figure $3(\mathrm{e})$ by blowing up the point represented by the corners of the rectangle and then blowing down the fiber through it. Figure $3(\mathrm{f})$ is the same as Figure 2(a) except that $\mathcal{F}_{2}$ is cut along another fiber. 
Proposition 1.2. The arrangements $\mathcal{A}^{\prime \prime}(5-b, b, 2), 0 \leq b \leq 3$, are realizable by real algebraic curves in the linear system $|4 H|$ on $\mathcal{F}_{2}$.

Proof. Follows from Lemma 1.1, see Figure 2(b,c).

Lemma 1.3. There exists a real algebraic curve in the linear system $|4 H|$ on $\mathcal{F}_{2}$ with a singular point of type $J_{10}$ (three tangent branches) arranged as in Figure 4(a) up to isotopy.

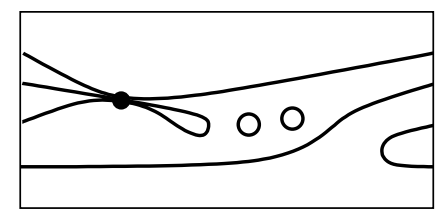

(a)

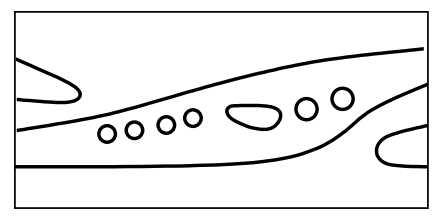

(b)

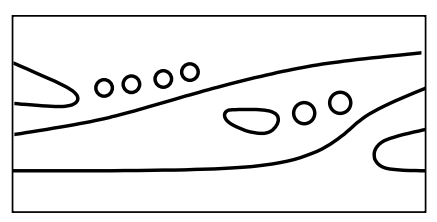

(c)

FiguRE 4

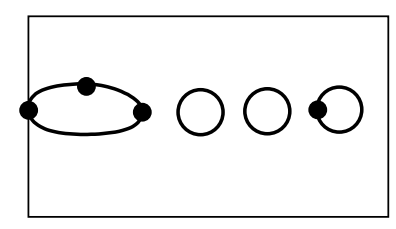

(a). $\mathcal{F}_{4}$

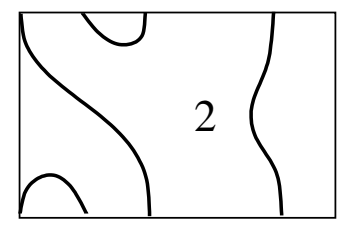

(b). $\mathbb{P}^{1} \times \mathbb{P}^{1}$

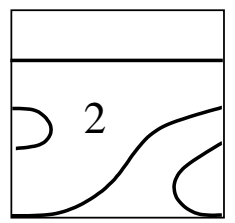

(c). $\mathbb{P}^{1} \times \mathbb{P}^{1}$

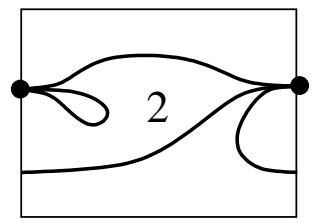

(d). $\mathcal{F}_{2}$

FiguRe 5

Proof. The construction is shown in Figure 5. We start with an $M$-curve of bidegree $(2,8)$ on $\mathcal{F}_{4}$ and we choose four points on it as in Figure 5(a). Then we blow up these points and blow down the fibers passing through them (see Remark 1.4). We obtain a curve of bidegree $(2,4)$ on $\mathbb{P}^{1} \times \mathbb{P}^{1}$ whose fiberwise arrangement with respect to the pencil induced from $\mathcal{F}_{4}$ is shown in Figure $5(\mathrm{~b})$. The fiberwise arrangement of this curve with respect to the other pencil is shown in Figure 5(c). Finally, we blow up twice the point corresponding to the corners of the rectangle in our picture and we blow down the fiber and the exceptional divisor of the first blowup. We obtain Figure $5(\mathrm{~d})$ which is the same as Figure $4(\mathrm{a})$ but $\mathcal{F}_{2}$ is cut along another fiber.

Remark 1.4. In terms of equations, the passage from Figure 5(a) to Figure 5(b) means the following. Let $y^{2}+a_{1}(x) y+a_{0}(x)=0, \operatorname{deg} a_{0}=8, \operatorname{deg} a_{1} \leq 4$, be the equation of the curve in Figure 5(a) where a standard coordinate system on $\mathcal{F}_{4}$ is chosen so that all the four marked points are on the axis $y=0$, hence $a_{0}(x)=b(x) c(x)$, where the $x$-coordinates of the marked points are the roots of $b(x)$. Then the equation of the curve in Figure $5(\mathrm{~b})$ is $b(x) y^{2}+a_{1}(x) y+c(x)=0$.

Proposition 1.5. The arrangements $\mathcal{A}^{\prime \prime}(4,3,0)$ and $\mathcal{A}^{\prime \prime}(0,7,0)$ are realizable by real algebraic curves in the linear system $|4 H|$ on $\mathcal{F}_{2}$.

Proof. Follows from Lemma 1.3, see Figure 4(b,c).

Lemma 1.6. There exists a real algebraic curve in the linear system $|4 H|$ on $\mathcal{F}_{2}$ with singular points of types $D_{6}, D_{4}, A_{1}$ arranged as in Figure $6(a)$ up to isotopy. 


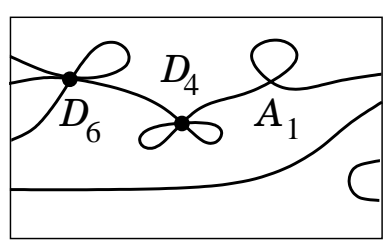

(a)

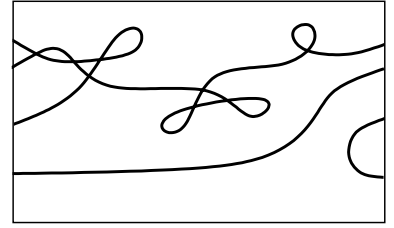

(b)

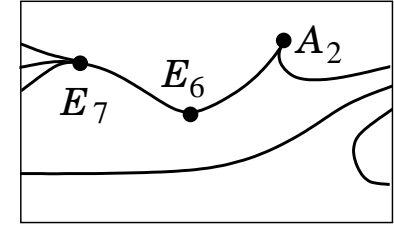

(c)

Figure 6

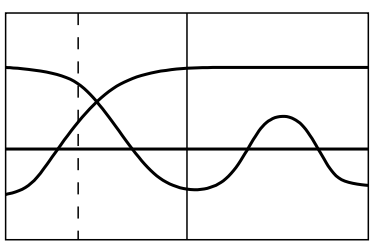

(a). $\mathcal{F}_{2}$

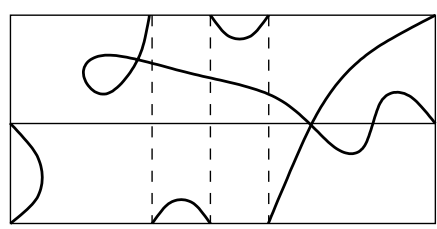

(d). $\mathbb{P}^{1} \times \mathbb{P}^{1}$

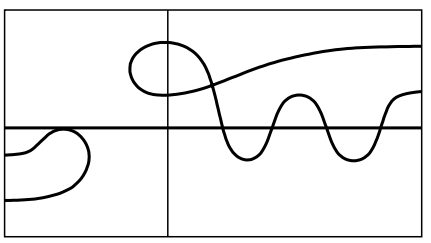

(b). $\mathcal{F}_{3}$

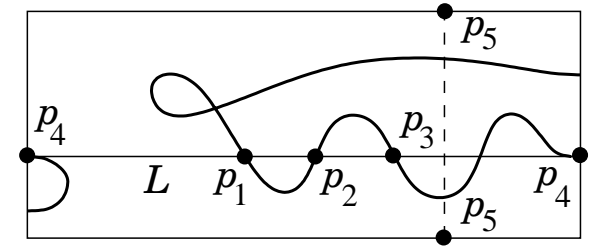

(c). $\mathcal{F}_{3}$

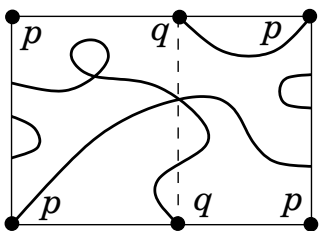

(e). $\mathbb{P}^{1} \times \mathbb{P}^{1}$

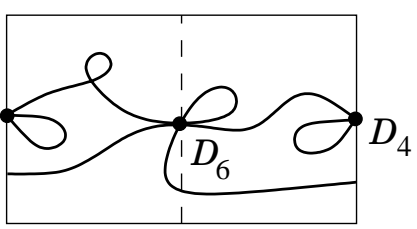

(f). $\mathcal{F}_{2}$

FIGURE 7

Proof. By gluing two reducible cubic curves on $\mathcal{F}_{1}$ we obtain a reducible curve on $\mathcal{F}_{2}$ shown in Figure $7(\mathrm{a})$. The gluing can be understood either in the sense of [21] or in the sense of [30]. Then we cut Figure 7(a) along the dashed line and glue it with another cubic curve as shown in Figure 7(b). We obtain a reducible curve in $\mathcal{F}_{3}$. The same curve is shown in Figure $7(\mathrm{c})$ but $\mathcal{F}_{3}$ is cut along another fiber. It is the union of a curve $L$ of bidegree $(1,3)$ and a nodal curve $C$ of bidegree $(2,6)$. We blow up the points $p_{1}, \ldots, p_{5}$ (see Figure $7(\mathrm{c})$ ) and then we blow down the fibers passing through them. We obtain a reducible curve on $\mathbb{P}^{1} \times \mathbb{P}^{1}$ shown in Figure $7(\mathrm{~d})$. The same arrangement is shown in Figure $7(\mathrm{e})$ but the coordinates are swapped. Finally, we blow up the points $p$ and $q$ and we blow down the (vertical) fibers passing through them. We obtain a curve in the linear system $|4 H|$ on $\mathcal{F}_{2}$ shown in Figure $7(\mathrm{f})$. It is the same curve as in Figure 6(a) but $\mathcal{F}_{2}$ is cut along another fiber.

Remark 1.7 The proof of Lemma 1.6 can be easily modified to obtain the curve shown in Figure 6(c).

Proposition 1.8. The arrangement $\mathcal{A}^{\prime}(3,3,1)$ is realizable by a real algebraic curve in the linear system $|4 H|$ on $\mathcal{F}_{2}$.

Proof. Follows from Lemma 1.6, see Figure 6(b).

\section{Restrictions on PSEUdoholomorphic CURVES}

2.1. Classification of smooth pseudoholomorphic curves: Proof of Theorem 1(b). The case of $M$ - and $(M-1)$-curves is done in [25]. Since any empty 
oval of a real pseudo-holomorphic curve can be removed, it is enough to exclude the following arrangements:

$$
\begin{aligned}
& \mathcal{A}^{\prime}(1,1,4), \mathcal{A}^{\prime}(2,1,3), \mathcal{A}^{\prime}(1,2,3), \\
\mathcal{A}^{\prime \prime}(5,1,1), & \mathcal{A}^{\prime \prime}(4,2,1), \mathcal{A}^{\prime \prime}(5,2,0), \mathcal{A}^{\prime \prime}(1,5,1), \mathcal{A}^{\prime \prime}(1,6,0) .
\end{aligned}
$$

The corresponding braids contradict Murasugi-Tristram inequality (see [19; §3.1])

$$
\operatorname{Null}_{\zeta}(b) \geq\left|\operatorname{Sign}_{\zeta}(b)\right|+4-e(b), \quad \zeta=\exp (2 \pi i \theta)
$$

for $\theta=1 / 2,1 / 2,1 / 2,1 / 5,1 / 5,2 / 7,1 / 5,2 / 7$ respectively.

\subsection{Restrictions on singular pseudoholomorphic curves needed for the proof of Theorem 1(a).}

Lemma 2.1. Let $C$ be a nodal pseudoholomorphic curve on $\mathcal{F}_{2}$ homologous to $4 H$ such that $\mathbb{R} C$ is arranged as shown in Figure $8(a)$ or (b). Then $C$ is reducible, more precisely, $C=C_{1} \cup C_{2}$ where $C_{1}$ and $C_{2}$ are pseudo-holomorphic curves homologous to $2 H$, moreover, $C_{1}$ (shown by solid line) is irreducible and $C_{2}$ (shown by dashed line) may or may not be reducible.

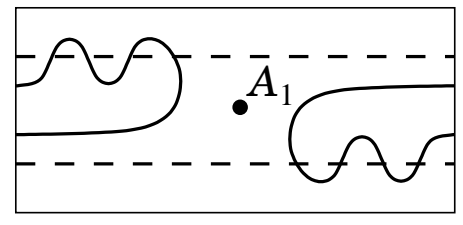

(a)

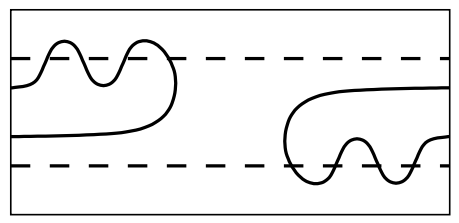

(b)

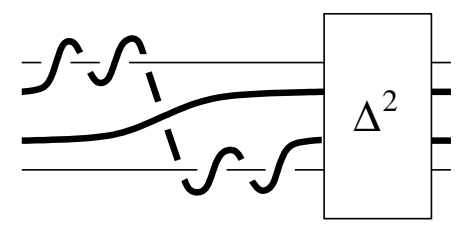

(c)

Figure 8

Proof. It is enough to prove this fact for Figure 8(b). Without loss of generality we may assume also that all non-real points of $C$ are smooth. We use the notation similar to that in $[19 ; \S 4.5]$. Let $(x, y)$ be standard coordinates on $\mathcal{F}_{2}$ in the chart corresponding to Figure 8, such that the projection pr $: \mathcal{F} \rightarrow \mathbb{P}^{1}$ is given by $(x, y) \mapsto x$. Let $H$ be the half of $\mathbb{C} P^{1} \backslash \mathbb{R} P^{1}$ given by $\operatorname{Im} x>0$ and $N=C \cap \operatorname{pr}^{-1}(H)$. Let $H^{-}$be a closed disk in $H$ which contains all the branch points of pr $\left.\right|_{N}$ and let $S^{3}$ be the boundary of a bidisk $\operatorname{pr}^{-1}\left(H^{-}\right) \cap\{|y| \leq R\}, R \gg 1$. We set $L=S^{3} \cap N$. So, the link $L$ in $S^{3}$ is the closure of the braid $b$ shown in Figure 8(c).

Let $\mu_{L}$ and $\mu_{N}$ be the number of components of $L$ and $N$ respectively and let $g$ be the total genus of $N$. Since $b$ is quasipositive, we have

$$
1=4-e(b)=\chi(N)=2 \mu_{N}-2 g-\mu_{L}=2 \mu_{N}-2 g-3
$$

where $e(b)$ is the exponent sum of $b$ and $\chi(N)$ is the Euler characteristic of $N$. Thus we have $\mu_{N}=2+g \geq 2$.

Let $L_{1}$ be the sublink of $L$ formed by the two central strands (the bold line in Figure 8(c)) and $L_{2}=L_{2}^{\prime} \sqcup L_{2}^{\prime \prime}=L \backslash L_{1}$. The linking numbers are: $\operatorname{lk}\left(L_{1}, L_{2}^{\prime}\right)=$ $\operatorname{lk}\left(L_{1}, L_{2}^{\prime \prime}\right)=0$ and $\operatorname{lk}\left(L_{2}^{\prime}, L_{2}^{\prime \prime}\right)=2$. Combined with the inequality $\mu_{N} \geq 2$, this fact implies that $N$ has two connected components $N_{1}$ and $N_{2}$ and $S^{3} \cap N_{i}=L_{i}, i=1,2$. 
Hence the closure $C_{i}$ of $N_{i} \cup \operatorname{Conj}\left(N_{i}\right), i=1,2$, is a smooth pseudoholomorphic curve.

Remark 2.2. The arrangements in Figure $8(\mathrm{a}, \mathrm{b})$ are realizable by real pseudoholomorphic curves on $\mathcal{F}_{2}$ homologous to $4 H$. Indeed, the braid corresponding to Figure $8(\mathrm{a})$ is $b=\sigma_{3}^{-4} \sigma_{2}^{-2} \sigma_{1}^{-4} \Delta^{2}$. It is quasipositive: $b=\left(a_{1}^{-1} \sigma_{2} a_{1}\right)\left(a_{2}^{-1} \sigma_{1} a_{2}\right)$ where $a_{1}=\sigma_{1}^{-1} \sigma_{3}^{-1} \sigma_{2} \sigma_{3}^{4}, a_{2}=\sigma_{2} \sigma_{3}^{3}$.

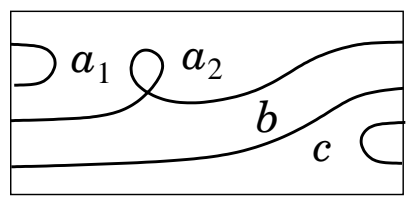

(a)

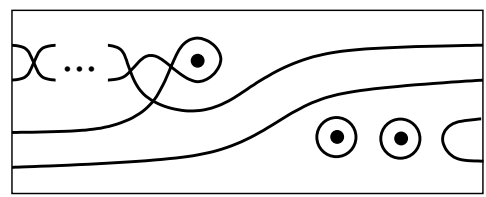

(b)

\section{FIGURE 9}

Lemma 2.3. If $a_{2} \geq 1, c \geq 1$, and $b+c \geq 2$, then the fiberwise arrangement in Figure $9\left(\right.$ a) is unrealizable by a real pseudoholomorphic curve on $\mathcal{F}_{2}$ homologous to $4 H$.

Proof. If there exists a real pseudoholomorphic curve as in Figure 9(a), then any two consecutive ovals in the same region can be joint by a node because this operation does not change the corresponding braid. Thus a curve which contains a subset shown in Figure 9(b) is also realizable (here we suppose that $c \geq 2$, otherwise one of the lower ovals should be moved to the central region). This arrangement contradicts Bezout's theorem for the auxiliary curve homologous to $H$ and passing through the three marked points.

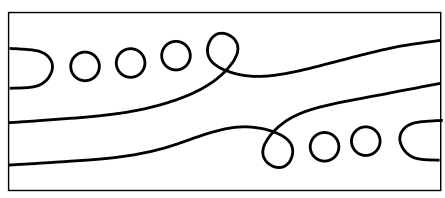

(a)

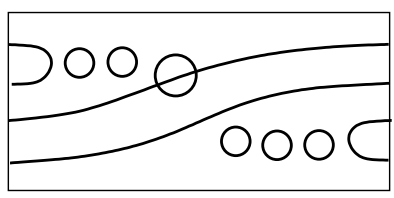

(b)

FIGURE 10

Lemma 2.4. The fiberwise arrangements in Figure 10(a,b) are unrealizable by real pseudoholomorphic curves on $\mathcal{F}_{2}$ homologous to $4 H$.

Proof. Follows from Murasugi-Tristram inequality (1) for $\theta=1 / 2$.

Lemma 2.5. Let $C$ be a smooth pseudo-holomorphic curve on $\mathcal{F}_{2}$ homologous to $3 H+2 F$ and transverse to $E$. Then a perturbation of $C+E$ cannot provide the almost fiberwise types listed in Theorem 1(b) but not in Theorem 1(a).

Proof. If $C+E$ can be smoothed out to $\mathcal{A}^{\prime \prime}(a, b, c)$, then it should be arranged as in Figure 11(a) up to exchange of $a$ and $c$. After blowing up $C \cap E$ followed by blowing down the strict transforms of the fibers (see Remark 1.4), such an arrangement transforms into a curve on $\mathcal{F}_{4}$ homologous to $3 H$ which can be smoothed out into Figure 11(b). Using the algorithm in [22], [24; §6], one easily checks (even by 


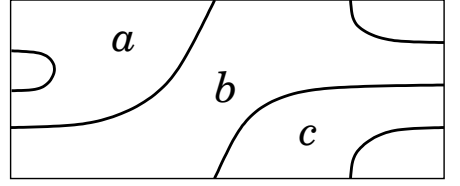

(a)

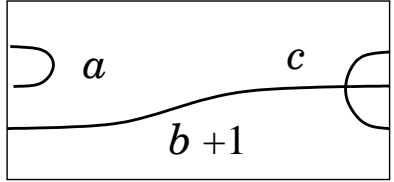

(b)

FIGURE 11

hand) that the corresponding 3-braids are not quasipositive. Since an erasing of an empty oval preserves pseudo-holomorphic realizability, it is enough to consider only $\mathcal{A}^{\prime \prime}(6,0,2)$ and $\mathcal{A}^{\prime \prime}(3,0,3)$.

It is easy to check (see also [2; Chapter 2, Section 15.2]) that all complex singularities of multiplicity 3 with $\delta$-invariant 6 or 7 are those in Table 1 up to homeomorphism ( $g$ is the genus of the Milnor fiber).

Table 1. Singularities of multiplicity 3 with $\delta=6$ or 7 .

\begin{tabular}{|c|c|c|c|c|c|c|c|}
\hline & equation & $g$ & $\delta$ & & equation & $g$ & $\delta$ \\
\hline$J_{10}$ & $y^{3}+x^{6}$ & 4 & 6 & $J_{12}$ & $\left(y+x^{2}\right)\left(y^{2}+x^{6}\right)$ & 5 & 7 \\
\hline$J_{11}$ & $\left(y+x^{2}\right)\left(y^{2}+x^{5}\right)$ & 5 & 6 & $J_{13}$ & $\left(y+x^{2}\right)\left(y^{2}+x^{7}\right)$ & 6 & 7 \\
\hline \multirow[t]{2}{*}{$E_{12}$} & $y^{3}+x^{7}$ & 6 & 6 & $E_{13}$ & $y\left(y^{2}+x^{5}\right)$ & 6 & 7 \\
\hline & & & & $E_{14}$ & $y^{3}+x^{8}$ & 7 & 7 \\
\hline
\end{tabular}

Let $p$ be an isolated singularity of a plane real analytic curve $C$. Let $B$ be a Milnor ball for $(C, p)$, i. e., the topology of $(B, C \cap B)$ does not change when the radius of $B$ tends to zero. Let $C^{\prime}$ be a smoothing of $C$ at $p$, that is a member of a real analytic family of curves $C_{t}, t \in[0, \varepsilon]$, such that $C_{0}=C$ and the topology of $\left(B, C_{t} \cap B\right)$ is constant on $\left.] 0, \varepsilon\right]$. Let $\alpha=b_{0}\left(\bar{C}^{\prime}\right)-1$ where $\bar{C}^{\prime}$ is obtained from the union of non-closed arcs of $C^{\prime}$ by identification of boundary points coming from a common real branch of $C$ (here $b_{0}$ stands for the number of connected components). Note that $b_{0}\left(\bar{C}^{\prime}\right)=b_{0}\left(\mathbb{R} C^{\prime} \cup \partial\left(C^{\prime} \cap B\right)\right)$. Let $l_{\text {loc }}$ be the number of ovals (closed components) of $C^{\prime}$. The local analog of Harnack bound (which is an immediate consequence of Smith-Thom inequality; see also [14, 27] and [13; Proposition 1]) states that

$$
l_{\mathrm{loc}} \leq g-\alpha
$$

where $g$ is the genus of $C^{\prime}$, i. e. the genus of the Milnor fiber of $(C, p)$. A similar bound takes place in the pseudoholomorphic setting.

Lemma 2.6. Let $C$ be a real pseudoholomorphic curve (maybe reducible but without multiple components) in $\mathcal{F}_{2}$ homologous to $4 H$ with a singular point $p$ of multiplicity 3 and with $\delta$-invariant 6 or 7 (see Table 1). Suppose that $E \not \subset C$. Let $F_{0}$ be a fiber such that $p \notin F_{0}$. Then $C$ cannot be smoothed out to a curve of almost fiberwise isotopy type $\mathcal{A}^{\prime \prime}(6,0,2)$ relative to $F_{0}$.

Proof. Singularities of pseudoholomorhic curves can be always smoothed out independently, so, we may assume that $p$ is the only singular point of $C$. Then $C$ is irreducible because the intersection of any local branch at $p$ with the the other branches is at most 5 whereas $C=C_{1}+C_{2}$ implies $C_{1} \cdot C_{2} \geq 6$. 


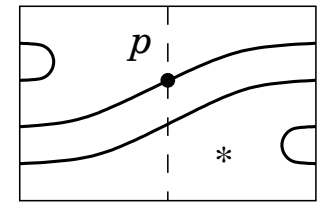

(a)

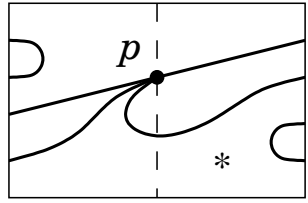

(b)

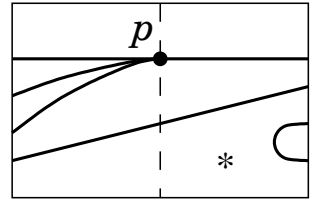

(c)

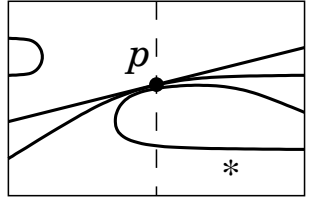

(d)

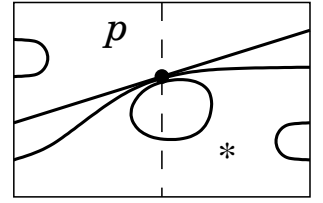

(e)

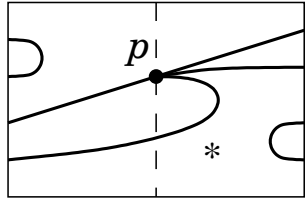

(f)

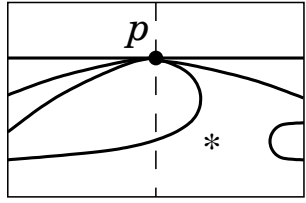

$(\mathrm{g})$

FIGURE 12

If $C$ admits a perturbation of type $\mathcal{A}$, then the union of the connected components of $\mathbb{R} C \backslash p$ which cross $F_{0}$, is necessarily one of those shown in Figure 12 where $F_{0}$ is represented by the vertical sides of the rectangles. Let $l(C)$ and $l\left(C^{\prime}\right)$ be the number of free ovals of $C$ and $C^{\prime}$ respectively and let $r(C)$ be the number of real branches of $C$ which cross $F_{0}$. So, we have $l(C)+r(C) \leq 10-\delta$.

For Figure $12(\mathrm{a})-(\mathrm{d})$, a smoothing of type $\mathcal{A}^{\prime \prime}(a, b, c)$ with $a c \neq 0$ is impossible. Indeed, the ovals appearing near $p$ cannot contribute to $c$ whereas no oval $v$ of $C$ can appear in the lower region to the right of $p$ (the region marked by the asterisk) by Bezout theorem for an auxiliary curve homologous to $H$, tangent to $C$ at $p$, and passing through $v$ (thus we exclude $E_{12}$ and $E_{14}$ ). The same argument shows that

(*) the order of tangency of the two lower arcs at $p$ is two

(hence $E_{13}$ is excluded as well).

Suppose that $(C, p)$ is of type $J_{11}$ or $J_{13}$. Due to $(*)$, we have $r(C)=2$ (see Figure 12(f)) whence $l(C) \leq 10-\delta-r(C)=8-\delta$ and we obtain $l\left(C^{\prime}\right) \leq l(C)+l_{\text {loc }} \leq$ $8-\delta+g=7$ by $(2)$, thus $C^{\prime} \notin \mathcal{A}(6,0,2)$.

Suppose now that $(C, p)$ is of type $J_{10}$ or $J_{12}$. Then we have $l(C) \leq 10-\delta-$ $r(C) \leq 9-\delta$. By $(2)$ this imlies $l\left(C^{\prime}\right) \leq l(C)+l_{\text {loc }}+1 \leq(9-\delta)+(g-\alpha)+1=8-\alpha$. Thus, if $C^{\prime} \in \mathcal{A}(6,0,2)$, then we have the equality sign in all these inequalities, i. e.,

$$
l\left(C^{\prime}\right)=l(C)+l_{\mathrm{loc}}+1, \quad r(C)=1, \quad \alpha=0 .
$$

The condition $r(C)=1$ combined with $(*)$ excludes already Figure 12(e,g). In the remaining case of Figure 12(f), we should consider:

(i) two ways of attaching the missing arc of $C$ (see Figure 13 on the left);

(ii) four ways to join the arcs of $C$ at $p$ (see Figure 13 on the right).
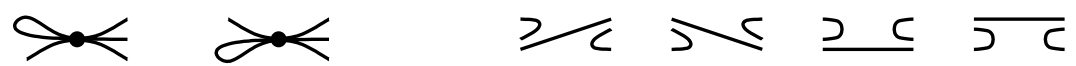

FigURE 13

It is easy to check that (3) is attained only for $J_{12}$ and only with the rightmost choices in (i) and (ii). However, in this case we obtain a curve of type $\mathcal{A}(a, b, c)$ with $b \geq 1$. 


\section{EXPlicit EQUATIONS OF SOME SINGULAR ALGEBRAIC CURVES}

3.1. Curves with singularities $A_{10}+A_{5}+A_{1}$ and $A_{9}+\left[A_{5}+A_{1}\right]$. Here we find explicit equations for all irreducible curves $C$ in the linear system $|4 H|$ on $\mathcal{F}_{2}$ with the following sets of singularities:

(i) $A_{10}+A_{5}+A_{1}$,

(ii) $A_{9}+\left[A_{5}+A_{1}\right]$

where the brackets in (ii) mean that $A_{5}$ and $A_{1}$ are on the same fiber of the fibration $\mathcal{F}_{2} \rightarrow \mathbb{P}^{1}$. The related Maple worksheet files are available on the web page http://www.math. univ-toulouse.fr/ orevkov/hrg3.html (supplied with detailed comments).

We use the computations from [6] which are based on the Artal-CarmonaCogolludo method [3] and on Moody's formulas for the Bertini involution [18].

Let $C \in|4 H|$ be a curve with an ordinary node at a point $p$. Let $F$ and $E$ be the fiber through $p$ and the exceptional section respectively. If we blow up $p$ and blow down $F$ and $E$, then we obtain a sextic curve $D$ on $\mathbb{P}^{2}$.

Let $(x, y)$ be a standard coordinate system on $\mathcal{F}_{2}$ such that $p$ is the point at infinity of the axis $y=0$ and let $f(x, y)=0$ be the equation of $C$. Then we can choose an affine coordinate chart on $\mathbb{P}^{2}$ so that $D$ is defined by the same equation $f(x, y)=0$. The Newton polygon of $f$ is contained in the quadrangle $Q=[(0,0),(6,0),(4,2),(0,4)]$. We have $Q=T_{1} \cap T_{2}$ where $T_{1}=[(0,0),(8,0),(0,4)]$ and $T_{2}=[(0,0),(6,0),(0,6)]$. So, $C$ and $D$ are the closures of the affine curve $f=0$ in the toric varieties corresponding to $T_{1}$ and $T_{2}$ respectively. Informally speaking, $f=0$ is an equation of $C$ (resp. of $D)$ if $Q$ is viewed as a subset of $T_{1}$ (resp. of $T_{2}$ ).

If $F$ transversally cuts $C$ outside $p$, then $D$ has an $A_{3}$ singularity. If the fiber $F$ contains an $A_{n}$ singularity, then it transforms into an $A_{n+4}$ singularity of $D$. Thus if $C$ satisfies (i) or (ii), then $D$ has a singularity set $A_{10}+A_{5}+A_{3}$ or $2 A_{9}$ respectively. To find all such curves, we adapt the computation for $A_{10}+A_{5}+A_{4}$ and $A_{10}+A_{9}$ which are given in detail in [6].

3.2. Sextic curves on $\mathbb{P}^{2}$ with singularities $A_{10}+A_{5}+A_{3}$. It is shown in $[6$; $\S 5.4]$ that all sextic curves with the set of singularities $A_{10}+A_{4}+A_{3}$ are contained in the two-parameter family (the parameters are denoted by $\mu$ and $\nu$ ) given by the following equation in $x_{1}, x_{2}, x_{3}$

$$
\phi_{6}-a\left(w-\mu w^{\prime}\right)^{2}=0
$$

where:

$$
\begin{aligned}
w & =x_{3}^{2}\left(a_{1} x_{1}+a_{2} x_{2}\right)+x_{3}\left(b_{1} x_{1}^{2}+b_{2} x_{1} x_{2}+b_{3} x_{2}^{2}\right)+\left(c_{1} x_{1}+c_{2} x_{2}\right) x_{1} x_{2}, \\
w^{\prime} & =x_{3}^{2}\left(a_{1}^{\prime} x_{1}+a_{2}^{\prime} x_{2}\right)+x_{3}\left(b_{1}^{\prime} x_{1}^{2}+b_{2}^{\prime} x_{1} x_{2}+b_{3}^{\prime} x_{2}^{2}\right)+\left(c_{1}^{\prime} x_{1}+c_{2}^{\prime} x_{2}\right) x_{1} x_{2}, \\
C_{5} & =\frac{A_{2}\left(B_{1}+\kappa x_{1} x_{3}^{2}\right)}{x_{2}}+\frac{\left(A_{1}-\kappa x_{1}^{2} x_{3}\right)\left(A_{2} x_{3}+B_{3} x_{2}\right)}{x_{2} x_{1}}+\kappa B_{3} x_{1} x_{3}, \\
\phi_{6} & =A_{1} C_{2}+x_{3} C_{5}, \quad \kappa=a_{1} b_{1}^{\prime}-a_{1}^{\prime} b_{1}, \\
A_{i} & =a_{i} w^{\prime}-a_{i}^{\prime} w, \quad B_{i}=b_{i} w^{\prime}-b_{i}^{\prime} w, \quad C_{i}=c_{i} w^{\prime}-c_{i}^{\prime} w, \quad i=1,2,3,
\end{aligned}
$$


the coefficients of $w$ and $w^{\prime}$ are

$$
\begin{aligned}
& a_{1}=\beta-\alpha, \\
& a_{2}=\alpha \beta-2 \alpha+1 \text {, } \\
& b_{1}=0 \text {, } \\
& b_{2}=\alpha \beta \rho-2 \alpha \rho+1 \text {, } \\
& b_{3}=\alpha-\alpha \rho, \\
& c_{1}=0 \text {, } \\
& c_{2}=\alpha \rho-\alpha \rho^{2} \text {, } \\
& a_{1}^{\prime}=\alpha \beta-2 \beta+1 \text {, } \\
& a_{2}^{\prime}=\alpha-\beta \text {, } \\
& b_{1}^{\prime}=\alpha \beta \rho-2 \beta \rho+1 \text {, } \\
& b_{2}^{\prime}=-2 \beta \rho+\alpha+\alpha \rho \text {, } \\
& b_{3}^{\prime}=0, \\
& c_{1}^{\prime}=\alpha \rho-\beta \rho^{2} \text {, } \\
& c_{2}^{\prime}=0 \text {, }
\end{aligned}
$$

and the parameters $a, \alpha, \beta$, and $\rho$ are expressed via $\mu$ and $\nu$ by:

$$
\begin{gathered}
\beta=\frac{\mu \nu \alpha+2 \mu \alpha+2 \nu \alpha-\mu-\nu+3 \alpha-2}{\mu \nu+\mu \alpha+\nu \alpha+2 \alpha-1}, \quad \rho=\frac{\mu \nu+\mu \alpha+\nu \alpha+2 \alpha-1}{(\mu+\alpha)(\nu+\alpha)}, \\
a=\frac{\alpha(\alpha-1)^{4}(\mu+\nu+2)(\mu-\nu)^{2}}{4(\mu+1)^{2}(\nu+2)(\mu+\alpha)^{3}(\nu+\alpha)^{2}} \\
\alpha=\frac{(\mu \nu-1)^{2}(\nu+2)}{4 \mu \nu^{2}+10 \mu \nu+\mu^{2}+5 \nu^{2}+8 \mu+12 \nu+8} .
\end{gathered}
$$

After the variable change

$$
x_{1}=z-x / \rho, \quad x_{2}=y+\frac{(\alpha \beta \rho-2 \beta \rho+1) x}{\rho(\beta \rho-\alpha)}, \quad x_{3}=x
$$

the curve $D$ defined by (4) has $A_{4}$ and $A_{10}$ singularities at $(0: 0: 1)$ and $(0: 1: 0)$ with the tangents $y=0$ and $z=0$ respectively and it has an $A_{3}$ singularity at

$$
\left(x_{1}: x_{2}: x_{3}\right)=\left(\frac{1}{1-\beta \rho}: \frac{1}{\rho-1}: \frac{1}{\beta-1}\right) .
$$

As it is explained in $[6 ; \S 5.4]$, a necessary condition for a further degeneration of $A_{4}$ to $A_{5}$ is the equation [6; (5.4)]. Being expressed via $\mu$ and $\nu$, it splits into four factors. Three of them correspond to the singularity sets $A_{10}+A_{4}+A_{3}+A_{1}$, $A_{10}+A_{4}+D_{4}, A_{10}+D_{5}+A_{3}$ and the fourth factor which is equal to the left hand side of

$$
\left(\mu^{2}+3 \mu\right) \nu^{2}+\left(4 \mu^{2}+15 \mu+7\right) \nu+2 \mu^{2}+10 \mu+6=0
$$

corresponds to $A_{10}+A_{5}+A_{3}$.

One can easily check that the equation (6) defines a rational curve in the plane $(\mu, \nu)$ which admits a parametrization

$$
\mu=-\frac{(2 t-1)(t-4)}{t^{2}-4 t+2}, \quad \nu=-\frac{3 t^{2}-10 t+4}{(2 t-1)(t-2)}
$$

Being expressed in $t$, the parameters $\alpha, \beta, \rho$, and $a$ take the form

$$
\alpha=\frac{4(2 t-1)(t-3)}{(5 t-3)(t-2)}, \quad \beta=\frac{t(t+1)}{2(t-3)(2 t-1)}, \quad \rho=\frac{2(5 t-3)(t-3)}{t^{2}},
$$




$$
a=\frac{81\left(t^{2}-4 t+2\right)^{2}\left(3 t^{2}-9 t+4\right)(t-1)^{2}(t-3)}{t^{5}(2 t-1)^{2}(t-2)^{2}} .
$$

So, we have found a one-parameter family of curves with the singularity set $A_{10}+A_{5}+A_{3}$. We have done it by solving a certain system of simultaneous equations on the coefficients. This family contains all sextic curves with the given set of singularities up to projective equivalence. To see it, one could trace all the computation and check that no solution is lost. However, this is not necessary because, due to [1; Theorem 2.5] (proven using the theory of K3 surfaces), the equisingular family $A_{10}+A_{5}+A_{3}$ corresponds to an irreducible curve in the moduli space.

3.3. Sextic curves on $\mathbb{P}^{2}$ with singularities $2 A_{9}$. Here we use the computations from $[6 ; \S 5.2]$. Namely, let $D$ be a curve given by (4), (5) where the non-zero coefficients of $w$ and $w^{\prime}$ are

$$
\begin{aligned}
& a_{1}=\beta-\alpha, \quad a_{2}=\alpha \beta-\alpha+\beta, \quad b_{2}=\beta-2 \alpha, \quad b_{3}=c_{3}=-\alpha, \\
& a_{1}^{\prime}=\alpha \beta+\alpha-\beta, \quad a_{2}^{\prime}=\alpha-\beta, \quad b_{2}^{\prime}=\alpha-2 \beta, \quad b_{1}^{\prime}=c_{1}^{\prime}=-\beta
\end{aligned}
$$

and

$$
\alpha=-\frac{(\mu+1)(\nu+1)}{(\mu+2)(\nu+2)}, \quad \beta=-\frac{(\mu+1)(\nu+1)}{\mu \nu+\mu+\nu+2} .
$$

Then, after the change of variables $x_{1}=z-x, x_{2}=y-x, x_{3}=x$, the curve $D$ has $A_{7}$ and $A_{9}$ singularities at $(0: 0: 1)$ and $(0: 1: 0)$ with the tangents $y=0$ and $z=0$ respectively. If

$a=-\frac{(\nu+1)^{2}(\nu+\mu+2)\left(\left(3 \mu^{2}+8 \mu+4\right) \nu^{2}+\left(8 \mu^{2}+22 \mu+12\right) \nu+4 \mu^{2}+12 \mu+7\right)}{4(\mu+1)(\mu+2)^{2}(\nu+2)^{3}(\mu \nu+\mu+\nu+2)^{2}}$

then the $A_{7}$ singularity degenerates to $A_{8}$. If, moreover, $\mu$ and $\nu$ satisfy the relation

$$
\left(\mu^{2}+6 \mu+4\right) \nu^{2}+\left(4 \mu^{2}+20 \mu+14\right) \nu+4 \mu^{2}+16 \mu+11=0,
$$

then $A_{8}$ further degenerates to $A_{9}$. The equation (7) defines the union of two rational curves in the plane $(\mu, \nu)$ which admit a parametrization

$$
\mu=-(t+2-\varepsilon) / t, \quad \nu=-(t-2) /(t-1), \quad \varepsilon= \pm \sqrt{5} .
$$

One can check that, for a generic $t$, the curve with $\varepsilon=\sqrt{5}$ is not projectively equivalent to any curve with $\varepsilon=-\sqrt{5}$. This can be done, for example, as follows. The choice of the coordinates ensures that the equation is determined by the curve up to rescaling of the variables. So, if we set the coefficients of $x^{6}, x^{5} y$, and $x^{5} z$ to be equal to 1 , then the equation is completely determined. Let $f_{t, \varepsilon}(x, y, z)=0$ be the equation normalized in this way and let $c_{i j}(t, \varepsilon)$ be its coefficients. It is enough to check that, for a fixed generic $t$, the simultaneous equations $c_{i j}(s,-\varepsilon)=c_{i j}(t, \varepsilon)$ on the indeterminate $s$ do not have any solution. Note, that the exchange of $y$ and $z$ transforms a curve with parameters $(t, \varepsilon)$ into a curve with the parameters $\left(\frac{3-\varepsilon}{2}-t, \varepsilon\right)$.

Thus, we have found two distinct one-parameter families in the moduli space of sextic curves. Due to [1; Proposition 2.6], the equisingularity stratum $2 A_{9}$ has two irreducible components. Thus we found all sextic curves with $2 A_{9}$ up to projective equivalence. 


\subsection{Arrangement of the singular curves on $\mathbb{R} \mathcal{F}_{2}$.}

Lemma 3.1. Let $C \in|4 H|$ be a real algebraic curve on $\mathcal{F}_{2}$ with singularities as in Section 3.1. Let $F_{0}$ be the fiber through $A_{5}$ and let $F_{1}$ be the fiber through $A_{9}$ or $A_{10}$. Then $C \cup F_{0} \cup F_{1}$ cannot be arranged up to isotopy as in Figure 14(a,b).

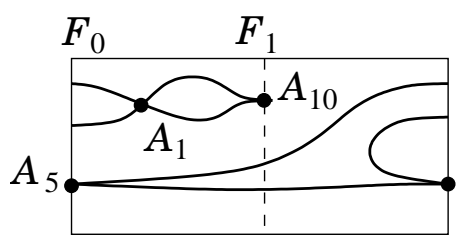

(a)

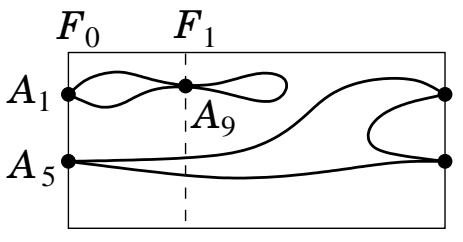

(b)

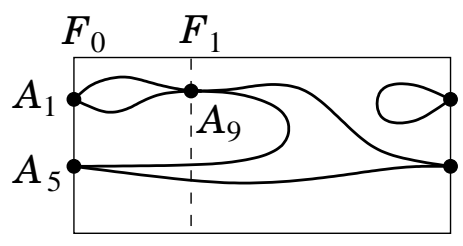

(c)

Figure 14

Proof. Case (i) in Section 3.1 (corresponds to Figure 14(a)). It is enough to check that the intersection points of $C \cup E$ with $F_{0}$ and $F_{1}$ cannot be arranged on $F_{0}$ and $F_{1}$ as in Figure 14. Indeed, if they are, then we have $d_{i}:=b_{i}^{2}-4 a_{i} c_{i}>0$ and $a_{i} c_{i}>0$ where $u^{2}\left(a_{i} u^{2}+b_{i} u+c_{i}\right)=0$ is the restriction of $C$ on the fiber $F_{i}$ (the coordinate $u$ on $F_{i}$ is chosen so that the singular point is at $u=0$ and the point $C \cap E$ is at $u=\infty)$.

After the transformation of $\mathcal{F}_{2}$ into $\mathbb{P}^{2}$ described in Section 3.1, the fiber $F_{0}$ (resp. $F_{1}$ ) corresponds to the line through $A_{3}$ and $A_{5}$ (resp. through $A_{3}$ and $A_{10}$ ). Using the formulas from Section 3.2, we check that $d_{0} a_{1} c_{1}=-11 R(t)^{2}$ where $R(t)$ is a rational function with integer coefficients. Thus $d_{0}$ and $a_{1} c_{1}$ cannot be positive simultaneously.

Case (ii) in Section 3.1 (corresponds to Figure 14(b)). Let $a_{1}, c_{1}, d_{1}$ be as in Case (i) and let $\delta_{n}, n=1,5$, be the discriminant of the restriction of the defining equation to the last exceptional curve of the resolution of the singular point $A_{n}$ (in fact, $\delta_{1}$ is the discriminant of the restriction of (4) to the line $z=0$ ). The local branches of $C$ at $A_{n}$ are real if and only if $\delta_{n}>0$. Thus, if $C$ is as in Figure 14(b), then $d_{1}, a_{1} c_{1}, \delta_{1}$, and $\delta_{5}$ are all positive. A computation based on the formulas from Section 3.3 shows that this condition never holds for $\varepsilon=-\sqrt{5}$ and it holds for $\varepsilon=\sqrt{5}$ only when $t \in] t_{1}, t_{2}[$, where

$$
t_{1}=(3-\sqrt{5}) / 2 \approx 0.38196, \quad t_{2}=(9-3 \sqrt{5}) / 4 \approx 0.57294
$$

(it happens that all roots of the polynomials involved belong to $\mathbb{Q}(\sqrt{5})$ ).

Let $f(x, y, z, t)$ be the left hand side of (4). Thus $f=0$ for a fixed $t$ is the equation of $D$. We consider the surface in $\mathbb{R P}^{2} \times \mathbb{R}$ defined by $f=0$. Let $x^{10} z^{8} \Delta(x, z, t)$ be the discriminant of $f$ with respect to $y$, i. e., $\Delta=0$ is the equation of the ramification locus of the projection of the surface $f=0$ onto $\mathbb{R P}_{(x: z)}^{1} \times \mathbb{R}_{(t)}$.

Let $a_{0}(t)$ be the coefficient of $y^{4} z^{2}$ in $f$ and let $\Delta_{1}(t)$ be the discriminant of $\Delta$ with respect to $x$. A straightforward computation shows that $a_{0}(t), \Delta_{1}(t)$, $\Delta(0,1, t)$, and $\Delta(1,0, t)$ do not have real roots in the interval $] t_{1}, t_{2}[$. This means that the fiberwise isotopy type of $C \cup F_{0} \cup F_{1} \cup E$ does not change when $t$ varies from $t_{1}$ to $t_{2}$. So, it is enough to establish this isotopy type for any value of $t$ belonging to this interval. A computation shows that it is as in Figure 14(c). 


\section{Background For the Hilbert-Rohn method}

To prohibit algebraic curves, we use a version of Hilbert-Rohn-Gudkov method (cf. $[25,26]$ ). Namely, we argue on the contrary assuming that an algebraic curve in question exists, showing that it must degenerate into a curve with certain singularities, and, finally, prohibiting the latter curve either by suitable methods of algebraic geometry and topology, or by a direct computation. We perform the degeneration process in several steps. In each step, we consider the stratum $S \subset|4 H|$ formed by real curves having certain isotopy type and specific singularities. Then we introduce a partial order on $S$ and show that, for any curve $C \in S$, there exists an equisingular family $C_{t} \in S, t \in[0, \varepsilon), C_{0}=C$, which is strongly monotone with respect to the introduced order. This yields that the extremal elements with respect to that order belong to $\bar{S} \backslash S$. Then we either go to the next step, considering the stratum of more degenerate curves, or show that no further degeneration is possible, hence deriving the desired contradiction to the existence of the initial curve.

Below we use the following notations: $\operatorname{Span}_{K}\{*\}$ denotes a linear space over the field $K$ with the indicated basis, $\langle *\rangle$ denotes an ideal in the corresponding algebra with the indicated generators.

4.1. Local geometry of equisingular families of curves. In this section, we describe the local geometry of equisingular families of curves that are needed for the proof of Proposition 5.1, which is the final step in the proof of the main Theorem 1. We notice that Lemmas 4.1 and 4.2 below are similar to [26; Propositions 2.1 and 2.4]. However, in the cited statements there is an incompleteness: namely, the upper bound to the number of real intersections of close singular curves was implicitly used, but not proved. Here we do prove the required bound (see Lemma 4.1(3)), thereby closing a gap in [26]. Furthermore, we slightly modify the definition of certain equisingular families in order to get a simpler proof of the bound (16). We supply a complete argument for the reader's convenience.

Let $\Sigma$ be a smooth algebraic surface, $(C, p) \subset(\Sigma, p)$ a curve germ with singularity of type $A_{2 s-1}, s \geq 1$, at $p \in \Sigma$, and let $U$ be its regular neighborhood (i.e. an open ball centered at $p$ such that $(C, p)$ is locally closed in $U$, is smooth outside $p$, and transversally intersects the boundary of any ball contained in $U$ and centered at $p$. Denote by $\mathcal{O}(\bar{U})$ the ring of continuous functions in $\bar{U}$ that are holomorphic in $U$. It has the maximal ideal $\mathfrak{m}=\{g \in \mathcal{O}(U) \mid g(p)=0\}$, and a norm $\|g\|_{\bar{U}}=\max |g(\bar{U})|$. Fix also a closed ball $V \subset U$ centered at $p$.

Fix a local coordinate system $S=(x, y)$ in $U$ so that $p=(0,0)$ and $(C, p)=$ $\left\{f:=y^{2}-x^{2 s}=0\right\}$. Let $\Lambda \subset \mathcal{O}(\bar{U})$ be a finite-dimensional linear space. Denote by $M_{C, p}^{\text {fix }}(\Lambda) \subset f+\Lambda$ the germ at $f$ of the affine subspace of $\mathcal{O}(\bar{U})$ defined by the vanishing of the coefficients of $x^{i} y^{j}$ below the Newton diagram of $f$. It is easy to see that $M_{C, p}^{\text {fix }}(\Lambda)$ does not depend on the choice of $S$. Denote by $M_{C, p}(S, \Lambda) \subset f+\Lambda$ the family of elements $g \in f+\Lambda$, representable as

$$
\left(\left(y^{\prime}\right)^{2}-\left(x^{\prime}\right)^{2 s}\right)\left(1+\varphi_{2}\left(x^{\prime}, y^{\prime}\right)\right)
$$

where

$$
\begin{gathered}
x^{\prime}=(x-\alpha)\left(1+\varphi_{3}(x, y)\right), \quad y^{\prime}=y-\left(x^{\prime}\right)^{s-1}\left(\beta+\varphi_{1}\left(x^{\prime}, y\right)\right), \\
\alpha, \beta \in \mathbb{C}, \varphi_{1} \in \mathfrak{m}, \varphi_{2}, \varphi_{3} \in \mathcal{O}(\bar{U}), \quad \text { and } \quad|\alpha|,|\beta|,\left\|\varphi_{1}\right\|_{\bar{U}},\left\|\varphi_{2}\right\|_{\bar{U}},\left\|\varphi_{3}\right\|_{\bar{U}}<\varepsilon
\end{gathered}
$$


where $0<\varepsilon \ll 1$ is chosen so that, for any given $\alpha, \beta, \varphi_{1}, \varphi_{2}, \varphi_{3}$ satisfying (10), formulas (9) determine a diffeomorphism such that the image of $U$ contains $V$ and the image of $p$ belongs to the interior of $V$.

Since, for any given $\alpha, \beta, \varphi_{1}, \varphi_{2}, \varphi_{3}$ satisfying (10), formulas (9) determine a local diffeomorphism $\Phi(x, y)=\left(x^{\prime}, y^{\prime}\right)$, each element $g \in M_{C, p}(S, \Lambda)$ defines a curve germ $g=0$ with singularity $A_{2 s-1}$ at the point $(\alpha, 0)=\Phi^{-1}(0,0)$. If $Q_{1}, Q_{2}$ are the components of $(C, p)$, then each curve $\{g=0\} \subset U, g \in M_{C, p}(S, \Lambda)$, has also two components $Q_{1}^{\prime}, Q_{2}^{\prime}$ (intersecting with multiplicity $s$ ) such that $Q_{i}^{\prime}$ is close to $Q_{i}, i=1,2$. Notice also that $M_{C, p}(S, \Lambda)$ is a germ of an analytic subspace in $f+\Lambda$. Indeed, $M_{C, p}(S, \Lambda)$ can be regarded as a germ of the equisingular stratum in $\Lambda$ with an additional restriction that the order of elements of $M_{C, p}(S, \Lambda)$ on an arc $y-\beta(x+\alpha)^{s-1}$ at the point $(-\alpha, 0)$ is $\geq 2 s$ for some $\alpha, \beta \in \mathbb{C}$. The equisingular stratum is a smooth subvariety of any linear versal deformation base (see for example, [10, Theorem II.2.38(3)]). Identifying a versal deformation with a finite-dimensional subspace of $\mathcal{O}(\bar{U})$ that contains $\Lambda$, we obtain an equisingular stratum in $\Lambda$ as the intersection of a smooth analytic variety germ with a linear space. At last, the intersection conditions with arcs specified above, by elimination theory, are expressed as analytic equations to coordinates in $\Lambda$.

Lemma 4.1. In the above notations, suppose that $\Lambda$ surjectively projects onto $J_{\Sigma, p}^{2 s}:=\mathcal{O}(\bar{U}) / \mathfrak{m}^{2 s+1}$. Then:

(1) The germ of $M_{C, p}^{\mathrm{fix}}(\Lambda)$ at $f$ is the germ of an affine subvariety of $f+\Lambda$ of codimension $3 s$, which can be described in the above coordinate system $S=(x, y)$ as

$$
M_{C, p}^{\mathrm{fix}}(\Lambda)=\left\{g \in f+\Lambda \mid \operatorname{ord}_{Q_{i}}(g) \geq 2 s, i=1,2\right\}=\left\langle y^{2}, y x^{s}, x^{2 s}\right\rangle .
$$

(2) The germ of $M_{C, p}(S, \Lambda)$ at $f$ is a smooth subvariety of $f+\Lambda$ of codimension $3 s-2$ with tangent space

$$
T_{f} M_{C, p}(S, \Lambda)=\left\{g \in \Lambda \mid \operatorname{ord}_{Q_{i}}(g) \geq 2 s-1, i=1,2\right\}=\left\langle y^{2}, y x^{s-1}, x^{2 s-1}\right\rangle .
$$

(3) Let $(C, p)=\left\{f:=y^{2}-x^{2 s}=0\right\}$ be real, $M \subset M_{C, p}(S, \Lambda)$ be the germ at $f$ of a smooth real one-dimensional analytic variety with $T M=\operatorname{Span}_{\mathbb{R}}\left\{f^{*}\right\}$, where $f^{*}=2 s \alpha_{0} x^{2 s-1}-2 \beta_{0} x^{s-1} y+\varphi(x, y)$ with $\varphi(x, y) \in\left\langle y^{2}, y x^{s}, x^{2 s}\right\rangle$, and let $(\mathbb{R}, 0) \rightarrow M, t \mapsto C^{(t)}$, be a regular parametrization with $C^{(0)}=C$ and $|t|<\varepsilon_{1} \ll 1$. Suppose that

$$
\left(C^{*} \cdot C\right)_{p}=4 s-2 \quad \text { where } \quad C^{*}=\left\{f+f^{*}=0\right\}
$$

and

$$
\left(C^{(t)} \cdot C\right)_{U}=4 s-2, \quad t \neq 0 .
$$

Then there exists $\varepsilon_{2}<\varepsilon_{1}$ such that for any $t, 0<|t|<\varepsilon_{2}$, first,

$$
\left(Q_{1}^{(t)} \cdot Q_{1}\right)_{\mathbb{R} U} \equiv\left(Q_{2}^{(t)} \cdot Q_{2}\right)_{\mathbb{R} U} \equiv s-1 \bmod 2,
$$

where $Q_{i}^{(t)}, i=1,2$, are the local branches of $C^{(t)}$ at its singular point in $U$; second, under additional assumptions $\alpha_{0} \neq 0$ and $s^{2} \alpha_{0}^{2} \neq \beta_{0}^{2}$, we have

$$
\#\left[\left(\left(Q_{1}^{(t)} \cap Q_{1}\right) \cup\left(Q_{2}^{(t)} \cap Q_{2}\right)\right) \cap \mathbb{R} U\right] \leq 2 .
$$


Proof. The first statement easily follows from the observation that $M_{C, p}^{\mathrm{fix}}(\Lambda)$ is the germ at $f$ of the linear subspace in $\mathcal{O}(U)$ defined by the vanishing of the coefficients of $x^{i} y^{j}$ below the Newton diagram of $f$.

For the second statement, we, first, notice that, in the case of singularity $A_{1}$, $M_{C, p}(S, \Lambda)$ is just a germ of an equisingular stratum, which is well-known to be smooth of codimension one. Suppose that $s>1$ and introduce the projection $M_{C, p}(S, \Lambda) \rightarrow\left(\mathbb{C}^{2}, 0\right), g \mapsto(\alpha, \beta)$. It is well-defined, since, for different $\alpha$ 's we get different positions of the singular point, and, for different $\beta$ 's, we get different positions of of the $(s-1)$-th infinitely near point to the singularity. Observe that the projection represents $M_{C, p}(S, \Lambda)$ as a locally trivial (topological) fibration over $\left(\mathbb{C}^{2}, 0\right)$ with fibers isomorphic to $M_{C, p}^{\text {fix }}(\Lambda)$, since,

(i) for fixed $\alpha$ and $\beta$, and for variable $\varphi_{1}, \varphi_{2}, \varphi_{3}$, formula (9) gives all function germs close to $\widetilde{f}\left(x^{\prime}, y^{\prime}\right)=f\left(x^{\prime}+\alpha, y^{\prime}-\beta\left(x^{\prime}\right)^{s-1}\right)$ that define curve germs with a singularity $A_{2 s-1}$ at $(-\alpha, 0)$ and its $(s-1)$ infinitely near points in a position, fixed by $\widetilde{f}$

(ii) the space $\Lambda$ surjectively projects onto $J_{\Sigma, p^{\prime}}^{2 s}$ for each point $p^{\prime} \in \Sigma$ close to $p$. This yields the dimension statement, particularly,

$$
\operatorname{dim} M_{C, p}(S, \Lambda)=\operatorname{dim} M_{C, p}^{\mathrm{fix}}(\Lambda)+2 .
$$

On the other hand, $M_{C, p}(S, \Lambda)$ is a germ of an analytic space, and its Zariski tangent space, consisting of all first order infinitezimal deformations, can be identified with $\Lambda \cap\left\langle y^{2}, y x^{s-1}, x^{2 s-1}\right\rangle$ when writing formula (9) in the form

$$
\begin{aligned}
y^{2}-x^{2 s} & \left(1+2 s \varphi_{3}(x, y)\right)+\varphi_{2}(x, y)\left(y^{2}-x^{2 s}\right)+2 s \alpha x^{2 s-1}+2\left(\beta+\varphi_{1}(x, y)\right) y x^{s-1} \\
& +O\left(|\alpha|^{2}+|\beta|^{2}+\left\|\varphi_{1}\right\|_{U}^{2}+\left\|\varphi_{2}\right\|_{U}^{2}+\left\|\varphi_{3}\right\|_{U}^{2}\right) .
\end{aligned}
$$

Thus, the smoothnes of $M_{C, p}(S, \Lambda)$ follows from the preceding dimension formula and the relation

$$
\operatorname{dim} \frac{\Lambda \cap\left\langle y^{2}, y x^{s-1}, x^{2 s-1}\right\rangle}{\Lambda \cap\left\langle y^{2}, y x^{s}, x^{2 s}\right\rangle}=\operatorname{dim} \frac{\left\langle y^{2}, y x^{s-1}, x^{2 s-1}\right\rangle}{\left\langle y^{2}, y x^{s}, x^{2 s}\right\rangle}=2 .
$$

At last, we show that $T_{f} M_{C, p}(S, \Lambda)=\left\langle y^{2}, y x^{s-1}, x^{2 s-1}\right\rangle \cap \Lambda$ can equivalently be expressed by (12). Indeed, the generators $g$ of the ideal $\left\langle y^{2}, y x^{s-1}, x^{2 s-1}\right\rangle$ satisfy relations $\operatorname{ord}_{Q_{i}}(g) \geq 2 s-1, i=1,2$, indicated in (12). On the other hand, these relations mean that $\left.g(x, y)\right|_{y=x^{s}}=g\left(x, x^{s}\right)=O\left(x^{2 s-1}\right)$ and $\left.g(x, y)\right|_{y=-x^{s}}=$ $g\left(x,-x^{s}\right)=O\left(x^{2 s-1}\right)$, which excludes germs outside the ideal $\left\langle y^{2}, y x^{s-1}, x^{2 s-1}\right\rangle$.

To prove formula (15), we, first, notice that (13) combined with (12) yields $\left(C^{*} \cdot Q_{i}\right)_{p}=2 s-1, i=1,2$. This implies by [12; Theorem 2] (see also [10; Lemma II.2.18]) that $\left(C^{(t)} \cdot Q_{i}\right)_{U} \geq 2 s-1, i=1,2$, which together with (14) gives $\left(C^{(t)} \cdot Q_{i}\right)_{U}=2 s-1, t \neq 0, i=1,2$. Now, in view of $\left(Q_{i}^{(t)} \cdot Q_{3-i}\right)_{U}=s, i=1,2$, we derive that $\left(Q_{i}^{(t)} \cdot Q_{i}\right)_{U}=s-1, t \neq 0, i=1,2$, and hence relation (15).

Comparing formulas (9) with the expression for $f^{*}$, we can represent $C^{(t)}$ as $\left(y^{\prime}\right)^{2}-\left(x^{\prime}\right)^{2 s}=0$, where

$$
\begin{aligned}
& x^{\prime}=\left(x-t \alpha_{0}\right)\left(1+t \psi_{3}(t, x, y)\right) \\
& y^{\prime}=y-\left(x^{\prime}\right)^{s-1}\left(\beta_{1} t(1+O(t))+x^{\prime} \psi_{1}\left(t, x^{\prime}, y\right)+y \psi_{2}\left(t, x^{\prime}, y\right)\right)
\end{aligned}
$$


with some fixed analytic functions $\psi_{1}, \psi_{2}, \psi_{3}$, and with $\beta_{1}=\beta_{0}-(s-1) \psi_{3}(0)$. Thus, we can represent the intersections $Q_{1}^{(t)} \cap Q_{1}$ and $Q_{2}^{(t)} \cap Q_{2}$ in $U$ as the solutions of the equations

$$
\begin{aligned}
& x^{s}=t \beta_{0}\left(x-t \alpha_{0}\right)^{s-1}+\left(x-t \alpha_{0}\right)^{s}+t\left(x-t \alpha_{0}\right)^{s} O(1), \\
& x^{s}=-t \beta_{0}\left(x-t \alpha_{0}\right)^{s-1}+\left(x-t \alpha_{0}\right)^{s}+t\left(x-t \alpha_{0}\right)^{s} O(1),
\end{aligned}
$$

respectively, where $O(1)$ is bounded from above by a positive function of $U, V$, $\varepsilon, \varepsilon_{1}, \alpha_{0}, \beta_{0}, \psi_{1}, \psi_{2}, \psi_{3}$, and, furthermore, we look for solutions that appear in a small neighborhood of zero. Note that such roots cannot have an asymptotics $x \sim \gamma t^{a}, t \rightarrow 0$, with $a<1$, since otherwise, in the above equations we would encounter the terms $\left(\beta_{0}-s \alpha_{0}\right) t^{1+(s-1) a},\left(\beta_{0}+s \alpha_{0}\right) t^{1+(s-1) a}$, respectively, with the minimal exponent of $t$ and which do not cancel out with any other term. So, in view of $\alpha_{0} \neq 0$ we rescale these equations by $x \mapsto t \alpha_{0} x$ and restrict attention to the only roots which stay in a finite interval as $t \rightarrow 0$, and thus, we end up with the two claims, which ensure the desired relation (16). If $\gamma \geq 0$, then

(i) for $s>0$ even, each of the equations

$$
x^{s}=\gamma(x-1)^{s-1}+(x-1)^{s}, \quad x^{s}=-\gamma(x-1)^{s-1}+(x-1)^{s}
$$

has one simple real solution and the others $s-2$ roots imaginary;

(ii) for $s>0$ odd, each of the equations (17) either has no real solutions, or two simple real solutions, or one double real solution, so that both equations have in total at most two real solutions (counting multiplicities).

Indeed, in the case of an even $s$, the claim (i) is evident for $\gamma=0$. The number of real roots might change in variation of $\gamma$ only when a multiple root occurs, i.e., the system

$$
\left\{\begin{array}{l}
x^{s}=\delta(x-1)^{s-1}+(x-1)^{s} \\
s x^{s-1}=\delta(s-1)(x-1)^{s-2}+s(x-1)^{s-1}
\end{array}\right.
$$

has a solution for some $\delta \in \mathbb{R}$. This system yields

$$
\delta=\frac{s(x-1)}{x-s}, \quad(x-s) x^{s}=x(x-1)^{s} .
$$

The solution $x=0, \delta=1$ is irrelevant, since the root $x=0$ of the corresponding equation

$$
x^{s}=(x-1)^{s-1}+(x-1)^{s} \Longleftrightarrow x^{s}=x(x-1)^{s-1}
$$

is simple. Another option, coming from the second equation in (19), is

$$
(x-s) x^{s-1}=(x-1)^{s} .
$$

It, however, has no real solutions: the left-hand side is negative as $x \in] 0, s[$, whereas the right-hand side is non-negative, and one has

$$
|x-s| \cdot|x|^{s-1}<|x-1|^{s} \quad \text { as } x \leq 0 \text { or } x \geq s,
$$

due to the inequality between the arithmetic and geometric mean for the numbers $|x-s|,|x|, \ldots,|x|$. 
Statement (ii) is evident for $s=1$. So, suppose that $s \geq 3$ is odd. Then, for $\gamma=0$, both equations (17) coincide and they have no real roots. Thus, we prove statement (ii) by showing that a multiple root of an equation $x^{s}=\delta(x-1)^{s-1}+$ $(x-1)^{s}$ occurs for a unique value of $\delta \in \mathbb{R}$, and this root is double. The search for a multiple root similarly reduces to system (18) and then to equations (19). Again the solution $x=0, \delta=1$ is irrelevant, and we similarly come up with the equation (21). Inequality (20) and the sign comparison restrict the search of a root to the interval ]0, 1[. Since the derivative $s x^{s-2}(x-s+1)$ of the left-hand side of (21) is negative in $] 0,1\left[\right.$, and the derivative $s(x-1)^{s-1}$ of the right-hand side of $(21)$ is positive in ]0,1[, we obtain a unique simple root. This completes the proof of claim (ii).

Lemma 4.2. Let $\Sigma$ be a smooth algebraic surface, $D$ an effective divisor class such that $-D K_{\Sigma} \geq 2$ and $H^{1}\left(\Sigma, \mathcal{O}_{\Sigma}\right)=0$. Let $C \in|D|$ be a reduced, irreducible curve having $r$ singular points $\mathbf{p}=\left\{p_{1}, \ldots, p_{r}\right\}$ of types $A_{2 k_{i}-1}, k_{i} \geq 1, i=1, \ldots, r$, and $s$ singular points $\mathbf{q}=\left\{q_{1}, \ldots, q_{s}\right\}$ of types $A_{2 l_{j}-1}, j=1, \ldots, s$, respectively, where $r, s \geq 0, r+s>0$. Denote by $M_{\mathbf{p}, \mathbf{q}}(C)$ the germ at $C$ of the family of curves $C^{\prime} \in|D|$, which, in a neighborhood $U\left(p_{i}\right)$ of any point $p_{i}, 1 \leq i \leq r$, are represented by $f_{i} \in M_{C, p_{i}}^{\text {fix }}(\Lambda)$ and, in a neighborhood $U\left(q_{j}\right)$ of any point $q_{j}$, $1 \leq j \leq s$, are represented by $g_{j} \in M_{C, q_{j}}(\Lambda)$, where $\Lambda=H^{0}(\Sigma, \mathcal{O}(m D))$ with a sufficiently large $m$.

If

$$
2 \sum_{i=1}^{r} k_{i}+2 \sum_{j=1}^{s}\left(l_{j}-1\right) \leq-D K_{\Sigma}-1
$$

then $M_{\mathbf{p}, \mathbf{q}}(C)$ is smooth of dimension

$$
d=g+\left(-D K_{\Sigma}-1-2 \sum_{i=1}^{r} k_{i}-2 \sum_{j=1}^{s}\left(l_{j}-1\right)\right)
$$

$g$ being the genus of $C$. Furthermore, if $d=1$ and $g=0$, and given a parametrization $(\mathbb{C}, 0) \rightarrow M_{\mathbf{p}, \mathbf{q}}(C), t \mapsto C^{(t)}, C^{(0)}=C$, and $C^{*} \in T_{C} M_{\mathbf{p}, \mathbf{q}}(C) \backslash\{C\}$, we have

$$
\left\{\begin{array}{llll}
\left(C^{*} \cdot C\right)_{p_{i}}=4 k_{i}, & p_{i} \in \mathbf{p}, & \left(C^{*} \cdot C\right)_{q_{j}}=4 l_{j}-2, & q_{j} \in \mathbf{q}, \\
\left(C^{(t)} \cdot C\right)_{p_{i}}=4 k_{i}, & p_{i} \in \mathbf{p}, & \left(C^{(t)} \cdot C\right)_{U\left(q_{j}\right)}=4 l_{j}-2, & q_{j} \in \mathbf{q}, t \neq 0 .
\end{array}\right.
$$

Proof. Due to Lemma 4.1, the smoothness and the dimension statement will follow from the transversality of the intersection of $\prod_{p_{i} \in \mathbf{p}} M_{C, p_{i}}(\Lambda) \times \prod_{q_{j} \in \mathbf{q}} M_{C, q_{j}}^{\text {fix }}(\Lambda) \subset$ $\Lambda^{r+s}$ with the image of $H^{0}\left(\Sigma, \mathcal{O}_{\Sigma}(D)\right)$ in $\Lambda^{r+s}$. This transversality is equivalent to the relation

$$
h^{0}\left(\Sigma, \mathcal{J}_{Z / \Sigma}(D)\right)=h^{0}\left(\Sigma, \mathcal{O}_{\Sigma}(D)\right)-\operatorname{deg} Z
$$

where $\mathcal{J}_{Z / \Sigma}$ is the ideal sheaf of the zero-dimensional scheme $Z \subset \Sigma$ defined in the points $p_{i} \in \mathbf{p}$ and $q_{j} \in \mathbf{q}$ by relations (12) and (11), respectively (each time $s$ is the $\delta$-invariant of the corresponding singularity). Since $h^{0}\left(Z, \mathcal{O}_{Z}\right)=\operatorname{deg} Z$ and in view of the exact cohomology sequence

$$
0 \rightarrow H^{0}\left(\Sigma, \mathcal{J}_{Z / \Sigma}(D)\right) \rightarrow H^{0}\left(\Sigma, \mathcal{O}_{\Sigma}(D)\right) \rightarrow H^{0}\left(Z, \mathcal{O}_{Z}\right) \rightarrow H^{1}\left(\Sigma, \mathcal{J}_{Z / \Sigma}(D)\right)
$$


associated to the exact sheaf sequence $0 \rightarrow \mathcal{J}_{Z / \Sigma}(D) \rightarrow \mathcal{O}_{\Sigma}(D) \rightarrow \mathcal{O}_{Z} \rightarrow 0$, we have to show that

$$
H^{1}\left(\Sigma, \mathcal{J}_{Z / \Sigma}(D)\right)=0 \text {. }
$$

From the exact sheaf sequence $0 \rightarrow \mathcal{O}_{\Sigma} \stackrel{\times C}{\longrightarrow} \mathcal{J}_{Z / \Sigma}(D) \rightarrow \mathcal{J}_{Z / C}(D) \rightarrow 0$ and the assumption $H^{1}\left(\Sigma, \mathcal{O}_{\Sigma}\right)=0$, we derive an exact sequence

$$
0=H^{1}\left(\Sigma, \mathcal{O}_{\Sigma}\right) \rightarrow H^{1}\left(\Sigma, \mathcal{J}_{Z / \Sigma}(D)\right) \rightarrow H^{1}\left(C, \mathcal{J}_{Z / C}(D)\right),
$$

which says that relation (25) follows from

$$
H^{1}\left(C, \mathcal{J}_{Z / C}(D)\right)=0 .
$$

To derive (26), we lift the sheaf $\mathcal{J}_{Z / C}(D)$ to the normalization $\nu: \widehat{C} \rightarrow C$. It is well-known (see, for example, [5; Section 2.4] or [7; Section 4.2.4]) that

$$
\nu_{*}\left(\mathcal{O}_{\widehat{C}}(-\Delta)\right)=\mathcal{J}_{C}^{\text {cond }}:=\operatorname{Ann}\left(\nu_{*} \mathcal{O}_{\widehat{C}} / \mathcal{O}_{C}\right)
$$

where $\Delta \subset \widehat{C}$ is the so-called double point divisor of degree $\operatorname{deg} \Delta=2 \sum_{z \in \operatorname{Sing}(C)} \delta(C, z)$. In our situation, the conductor ideal $\mathcal{J}_{C}^{\text {cond }}$ is defined at the points of $\mathbf{p} \cup \mathbf{q}$ by the ideals (cf. [7; Section 4.2.4])

$$
\begin{aligned}
& \mathcal{J}_{C, p_{i}}^{\text {cond }}=\left\{g \in \mathcal{O}_{C, p_{i}} \mid \operatorname{ord}_{P_{i, s}} g \geq k_{i}-1, s=1,2\right\}, \quad p_{i} \in \mathbf{p}, \\
& \mathcal{J}_{C, q_{j}}^{\text {cond }}=\left\{g \in \mathcal{O}_{C, q_{j}} \mid \operatorname{ord}_{Q_{j, s}} g \geq l_{j}-1, s=1,2\right\}, \quad q_{j} \in \mathbf{q},
\end{aligned}
$$

where $P_{i, 1}, P_{i, 2}$ are the branches of the germ $\left(C, p_{i}\right)$, and $Q_{j, 1}, Q_{j, 2}$ are the branches of the germ $\left(C, q_{j}\right)$. Comparing with formulas (12) and (11) applied to each point $p_{i} \in \mathbf{p}$ and $q_{j} \in \mathbf{q}$, respectively, we obtain that

$$
\mathcal{J}_{Z / C}(D)=\nu_{*} \mathcal{O}_{\widehat{C}}(\mathbf{d}-\Delta-\mathbf{w}),
$$

where divisors $\mathbf{d}, \mathbf{w} \subset \widehat{C}$ have degrees

$$
\operatorname{deg} \mathbf{d}=D^{2}, \quad \operatorname{deg} \mathbf{w}=2 \sum_{p_{i} \in \mathbf{p}} k_{i}+2 \sum_{q_{j} \in \mathbf{q}}\left(l_{j}-1\right) .
$$

Since

$$
\begin{aligned}
\operatorname{deg}(\mathbf{d}-\Delta-\mathbf{w}) & =D^{2}-2 \sum_{z \in \mathbf{p} \cup \mathbf{q}} \delta(C, z)-2 \sum_{p_{i} \in \mathbf{p}} k_{i}-2 \sum_{q_{j} \in \mathbf{q}}\left(l_{j}-1\right) \\
& =D^{2}-\left(D^{2}+D K_{\Sigma}+2-2 g\right)-2 \sum_{p_{i} \in \mathbf{p}} k_{i}-2 \sum_{q_{j} \in \mathbf{q}}\left(l_{j}-1\right) \\
& =2 g-2+\left(-D K_{\Sigma}-2 \sum_{p_{i} \in \mathbf{p}} k_{i}-2 \sum_{q_{j} \in \mathbf{q}}\left(l_{j}-1\right)\right) \stackrel{(22)}{>} 2 g-2,
\end{aligned}
$$

we, finally, obtain (26).

Relation (24) for the intersection of $C^{*}$ and $C$ immediately follows from (12) and Bézout theorem. In turn, relation (24) for intersection of $C^{(t)}, t \neq 0$, with $C$ follows again from Bézout theorem and inequalities

$$
\left(C^{(t)} \cdot C\right)_{U\left(p_{i}\right)} \geq\left(C^{*} \cdot C\right)_{p_{i}}, p_{i} \in \mathbf{p}, \quad\left(C^{(t)} \cdot C\right)_{U\left(q_{j}\right)} \geq\left(C^{*} \cdot C\right)_{q_{j}}, q_{j} \in \mathbf{q}
$$

(see [12; Theorem 2] or [10; Lemma II.2.18]). 
4.2. General position arguments for curves on $\mathcal{F}_{2}$. The following is the $\mathcal{F}_{2}$ analog of Brusotti's theorem on smoothing of real algebraic curves on $\mathbb{R P}^{2}$.

Lemma 4.3. Let $C \subset \mathcal{F}_{2}$ be a real nodal curve not containing the (-2)-curve $E$ (but possibly reducible). Then there exists a small real deformation of $C$ in the same linear system, which preserves prescribed conjugation-invariant set of nodes of $C$ (possibly moving them) and which smoothes out the remaining nodes so that, for any real smoothed node, one can prescribe one of the two possible smoothings.

Proof. This immediately follows from [9; Corollary 6.3], where the sufficient conditions for the linear system $|C|$ to be a joint versal deformation of all the nodes of $C$ are $-C^{\prime} K_{\mathcal{F}_{2}}>0$, where $C^{\prime}$ runs over all components of $C$. This inequality always holds as $C^{\prime} \neq E$.

Lemma 4.4. (1) No curve in the linear system $|H|$ can pass through 4 points in general position.

(2) No reduced curve in the linear system $|2 H|$ can have $r$ singular points and pass through s given points, all in general position, if $3 r+s>8$.

(3) No curve in the linear system $|3 H|$, which does not contain $E$ as component, can have 6 singular points so that 5 of them are in general position.

(4) The total $\delta$-invariant of a reduced curve in the linear system $|D|$ on $\mathcal{F}_{2}$ not containing neither $E$, nor any fiber as component does not exceed 6, 8, or 10 according as $D=3 H, 3 H+F$, or $4 H-E$.

(5) There are only finitely many curves in $|4 H|$ splitting into 4 distinct components from the linear system $|H|$ and having 6 singular points in general position. Furthermore, each of these curves has 12 nodes as its only singularities.

Here "in general position" means that there exists a Zariski open dense subset in the space of considered tuples such that each tuple in it possesses the required property.

Proof. (1) Follows from the relation $\operatorname{dim}|H|=3$.

(2) The family of reduced curves in $|2 H|$ having $r$ singularities has codimension $r$ in $|2 H|$. Indeed, without loss of generality, we can suppose that the singularities are simple nodes and then use, for instance, [9, Corollary 6.3]. The condition to pass thorugh additional $s$ generic points raises the codimension by $s$. Now, assuming that the projection of that family to $\left(\mathcal{F}_{2}\right)^{r}$, sending a curve to its singular points, is dominant (and this is just another way to say that the singular points are in general position), we obtain that $\operatorname{dim}|2 H|-(r+s)=8-r-s \geq 2 r$ or, equivalently, $3 r+s \leq 8$.

(3) We can rule out non-reduced curves, since then one deals with curves of type $C^{\prime}+2 C^{\prime \prime}, C^{\prime}, C^{\prime \prime} \in|H|$, and the latter curves can pass through at most three prescribed generic points. Next, we note that the reduced curves in $|3 H|$ having 6 singularities outside $E$ must split into three distinct components $C^{\prime}, C^{\prime \prime}, C^{\prime \prime \prime} \in|H|$ but such a curve may have $\leq 4$ singularities in general position by statement (1).

(4) The arithmetic genera of the divisors $3 H, 3 H+F$, and $4 H-E$ are 4,6 , and 8 , respectively. The hypotheses of the statement yield that the considered curves can split into at most three components, which implies the required bounds.

(5) All the components are smooth and they must provide at least 12 local branches at six singular points. On the other hand, since $\operatorname{dim}|H|=3$ and the six singular points are in general position, each of the four component hits at most 3 of 
them. Thus, each component passes through three of the six given singular points, and is uniquely defined by such a condition. This yields the required finiteness. Finally, the general position of the given singular points implies that all 12 pairwise intersection points of the components are distinct and transversal.

\section{Restrictions on Algebraic CURVES}

Proposition 5.1. There are no smooth real algebraic curves on $\mathcal{F}_{2}$ belonging to the linear system $|4 H|$ and having almost fiberwise isotopy types $\mathcal{A}^{\prime \prime}(6,0,2), \mathcal{A}^{\prime \prime}(4,0,3)$, $\mathcal{A}^{\prime \prime}(3,1,3), \mathcal{A}^{\prime \prime}(3,0,3)$.

The proof follows the lines of $[25,26]$ and uses a version of the Hilbert-RohnGudkov method based on the statements proved in Section 4.

5.1. Prohibition of curves of type $\mathcal{A}^{\prime \prime}(6,0,2)$. By [25; Lemma 3.3], the existence of a smooth curve of type $\mathcal{A}^{\prime \prime}(2,0,6)$ yields the existence of a real rational curve with 9 ordinary nodes of the almost fiberwise isotopy type shown in Figure 15(a). Denote this type by $\mathcal{A}_{9}^{\prime \prime}(6,0,2)$.

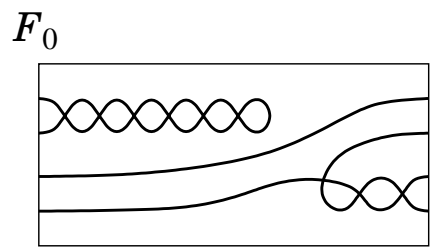

(a)

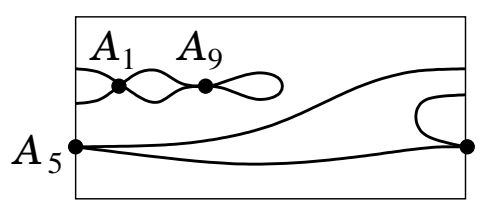

(b)

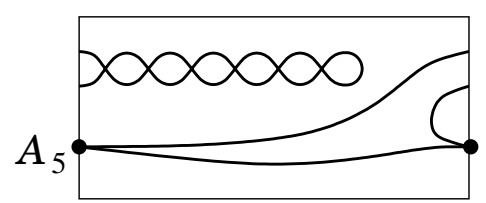

(c)

FiguRe 15

By [9; Theorem 6.1(ii,iii)], the family $\mathcal{R}$ of real rational nodal curves in the real part of $|4 H|$ is a smooth quasi-projective variety of codimension 9 . Denote by $\mathcal{R}\left(\mathcal{A}_{9}^{\prime \prime}(6,0,2)\right)$ the subfamily in $\mathcal{R}$ formed by curves of type $\mathcal{A}_{9}^{\prime \prime}(6,0,2)$.

We start with the following auxiliary statement, and then proceed with the Hilbert-Rohn-Gudkov method.

Lemma 5.2. A reduced, reducible real curve $C \in|4 H|$, not containing fibers as components and having only simple (i.e., $A-D-E$ ) singularities, cannot be a limit of a family of real rational curves from $\mathcal{R}\left(\mathcal{A}_{9}^{\prime \prime}(6,0,2)\right)$, whose singular points lie outside some fixed neighborhood of $E$.

Proof. Assume on the contrary that such a family does exist. We regard it as a deformation of a given reducible curve $C$. Clearly all components of $C$ are rational. It follows that irreducible simple singularities of $C$, i. e., $A_{2 k}, k \geq 1, E_{6}, E_{8}$, are deformed equigenerically (i. e., with the maximal possible number of nodes), since otherwise one would get a curve of a positive genus.

If a real singularity of $C$ has two complex conjugate local branches, then these branches belong to the same irreducible component of $C$, since otherwise one would have two complex conjugate components of $C$ non-trivially intersecting all fibers, and hence $C$ would not deform into a curve, intersecting infinitely many fibers at four real points. It then follows, that singularities of $C$ with a couple complex conjugate branches are deformed equigenerically.

Remaining singularities of $C$ may be of types $A_{2 k-1}, k \geq 1, D_{2 k}, k \geq 2$, or $E_{7}$, and their local branches all are real. We claim that, for these singularities, any 
local deformation with non-isolated real nodes as its only singularities factors (up to equivariant isotopy in a regular neighborhood) through a nodal equigeneric one, which then turns into the given nodal deformation by smoothing out certain nodes. Indeed, for singularities $A_{2 k-1}, k \geq 1$, and $D_{2 k}, k \geq 2$, this easily follows from classification of real nodal hyperelliptic curves. For singularity $E_{7}$ this follows from the known fact that any real rational nodal plane quartic curve with real nodes only can be obtained (up to isotopy) by smoothing out an intersection point of a real nodal cubic and a line (see, for instance, [11]). We also point out that, if $C \supset E$, then non-nodal singularities cannot lie on $E$, since otherwise one would get, in the considered deformation, some nodes in a neighborhood of $E$ contrary to the hypotheses of Lemma.

The given deformation of $C$ is nodal. On the other hand, by Lemma 6.2 in Section 6, any local deformations of singular points of $C$ can independently be realized by variation in the linear system $|4 H|$. In view of the above observation on deformations of simple singularities we derive that there exists a nodal reducible curve, which is a limit of real rational curves belonging to $\mathcal{R}\left(\mathcal{A}_{9}^{\prime \prime}(6,0,2)\right)$ and having singular points outside some fixed neighborhood of $E$. So, we may suppose that $C$ is nodal.

If $C \not \supset E$, the required statement follows from Lemma 2.3. Indeed, otherwise, we would have a family of rational nodal pseudoholomorphic curves of type shown in Figure 15(a) that converges to a pseudoholomorphic curve with an additional node, and hence splitting into two rational components. Since an irreducible pseudoholomorphic curve homologous to $k H, k \leq 3$, has at most 4 nodes, the newly appearing node in the considered degeneration must join one of the discs $d_{i}^{\prime}$ with the domain $d_{0}$ (see Figure 16(a)). Furthermore, here $i>1$, since otherwise, one would get only one real global one-dimensional branch contrary to the reducibility of the curve. However, this contradicts Lemma 2.3.

If $C \supset E$, then the required statement follows from Lemma 2.5.

Lemma 5.3. Suppose that $\mathcal{R}\left(\mathcal{A}_{9}^{\prime \prime}(6,0,2)\right) \neq \varnothing$. Then $\partial \mathcal{R}\left(\mathcal{A}_{9}^{\prime \prime}(6,0,2)\right)$ contains a real rational curve that has singularities $A_{1}, A_{5}$, and $A_{9}$ and the almost fiberwise isotopy type shown in Figure 15(b).

Proof. We proceed in two steps: first, show that a general curve in $\mathcal{R}\left(\mathcal{A}_{9}^{\prime \prime}(6,0,2)\right)$ can be degenerated into a curve shown in Figure 15(c), and, second, degenerate the obtained curve into the curve asserted in Lemma.

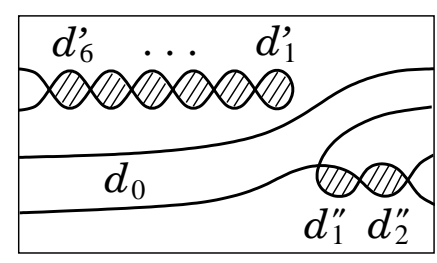

(a)

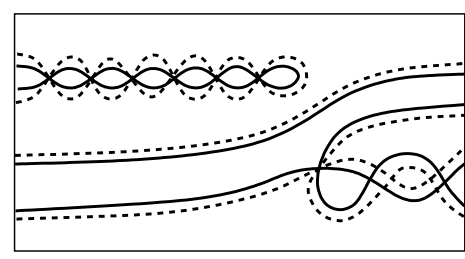

(b)

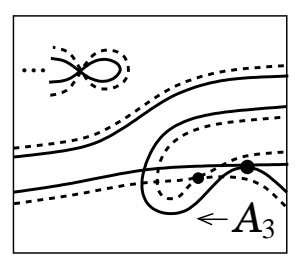

(c)

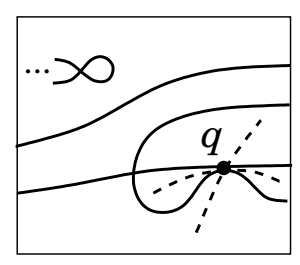

(d)

Figure 16

Step 1. For a curve $C \in \mathcal{R}\left(\mathcal{A}_{9}(6,0,2)\right)$, denote by $d_{0}(C), d_{i}^{\prime}(C), i=1, \ldots, 6$, and $d_{i}^{\prime \prime}(C), i=1,2$, the closed disks bounded by loops in $\mathbb{R} C$ and joined by singular points as marked by shadow in Figure 16(a). Introduce the partial order on $\mathcal{R}\left(\mathcal{A}_{9}(6,0,2)\right)$ setting $C \prec C^{\prime}$ if $d_{0}(C), d_{1}^{\prime}(C), \ldots, d_{6}^{\prime}(C), d_{1}^{\prime \prime}(C)$ are proper subsets 
of $d_{0}\left(C^{\prime}\right), d_{1}^{\prime}\left(C^{\prime}\right), \ldots, d_{6}^{\prime}\left(C^{\prime}\right), d_{1}^{\prime \prime}\left(C^{\prime}\right)$, respectively, and $d_{2}^{\prime \prime}\left(C^{\prime}\right)$ is a proper subset of $d_{2}^{\prime \prime}(C)$. For example, if $C$ (resp. $C^{\prime}$ ) is the curve shown by the solid line (resp. dashed line) in Figure 16(b), then we have $C \prec C^{\prime}$.

By [26; Proposition 2.4(b)] we can choose a curve $C \in \mathcal{R}\left(\mathcal{A}_{9}(6,0,2)\right)$ so that all its nodes, except for the two in $\partial d_{2}^{\prime \prime}(C)$, are in general position (here the required condition (6) from [26] reads $\left.2 \cdot 7<-C K_{\mathcal{F}_{2}}=16\right)$. Denote $\mathbf{p}=\operatorname{Sing}(C) \backslash d_{2}^{\prime \prime}(C)$ and $\mathbf{q}=\operatorname{Sing}(C) \cap d_{2}^{\prime \prime}(C)$. Let $S_{\mathbf{p}} \subset \mathcal{R}\left(\mathcal{A}_{9}(6,0,2)\right)$ be the stratum formed by curves whose 7 nodes are fixed at $\mathbf{p}$ and let us consider the germ of the family $M_{\mathbf{p}, \mathbf{q}}(C) \subset S_{\mathbf{p}}$ (see Lemma 4.2). Condition (22) of Lemma 4.2 is fulfilled, and hence $M_{\mathbf{p}, \mathbf{q}}(C)$ is a smooth one-dimensional real variety (cf. relation (23)). Furthermore, we derive from Lemma 4.1(3) that the curves $C^{\prime} \neq C$ in $M_{\mathbf{p}, \mathbf{q}}(C)$ intersect $C$ with multiplicity 4 at each node $z \in \mathbf{p}$ and intersect $C$ in a neighborhood of each node $z \in \mathbf{q}$ at two real points. It follows that there exists a connected component $M$ of $M_{\mathbf{p}, \mathbf{q}}(C) \backslash\{C\}$ such that the real part of curves $C^{\prime} \in M$ looks as shown by the dashed line in Figure 16(b), in particular, $C \prec C^{\prime}$.

This means that, for any curve $C \in S_{\mathbf{p}}$, the closed set $\sigma(C)=\overline{\left\{C^{\prime} \in S_{\mathbf{p}} \mid C \prec C^{\prime}\right\}}$ $\subset \bar{S}_{\mathbf{p}}$ is non-empty. Let us choose a continuous function $f: \sigma(C) \rightarrow \mathbb{R}$ which is strictly monotone for the partial order $\prec$. For example, we can set $f(C)=$ Area $\left(d_{i}^{\prime}(C)\right)$ for some $i=1, \ldots, 6$, or $f(C)=-$ Area $\left(d_{2}^{\prime \prime}(C)\right)$ where the area is defined by an arbitrary Riemannian metric on $\mathbb{R} \mathcal{F}_{2}$. Let $C_{0}$ be the element of $\sigma(C)$ where the maximum of $f$ is attained. It is clear that $C_{0} \in \bar{S}_{\mathbf{p}} \backslash S_{\mathbf{p}}$.

We claim that $C_{0}$ is irreducible. This would yield that the disk $d_{2}^{\prime \prime}\left(C_{0}\right)$ shrinks to a point, thus, $C_{0}$ has 7 nodes and a tacnode $A_{3}$ and the almost fiberwise isotopy type shown in Figure 16(c) by fat line. To prove the irreducibility of $C_{0}$, first, show that $C_{0}$ cannot contain a multiple component $C_{0}^{\prime}$ belonging to $|H|,|2 H|,|H+F|$, or $|H+2 F|$. Indeed, the intersection points of a current curve $C^{\prime} \in S_{\mathbf{p}}$ (tending to $\left.C_{0}\right)$ with any fiber crossing disks $d_{i}^{\prime}\left(C^{\prime}\right), i=1, \ldots, 6$, all are real and they move so that only two points are closing to each other, whereas the two other points run away towards $E$ from different sides. Hence the spoken component $C_{0}^{\prime}$ may belong only to $|H|,|H+F|$, or $|H+2 F|$, and its is smooth. The latter fact combined with the observation that the intersection point $C_{0}^{\prime} \cap F^{\prime}$ with any real fiber $F^{\prime}$ crossing the disc $d_{i}^{\prime}\left(C^{\prime}\right), 1 \leq i \leq 6$, lies below the interval $d_{i}^{\prime}\left(C^{\prime}\right) \cap F^{\prime}$ (see Figure 16(b)) yields that $C_{0}^{\prime}$ does not hit the six points of $\mathbf{p}$ lying on the boundary of the discs $d^{\prime}\left(C^{\prime}\right), 1 \leq i \leq 6$. Hence the remaining part of $C_{0}$, which belongs to $|2 H|,|H+E|$, or $|2 E|$, respectively, must have singularities at the aforementioned six points of $\mathbf{p}$ lying in general position. However, this is impossible (cf. Lemma 4.4). Suppose now that $C_{0}$ contains a double component $E$. Then the upper arcs of the disks $d_{i}^{\prime}\left(C^{\prime}\right), i=1, \ldots, 6$, of the current curve $C^{\prime} \in S_{\mathbf{p}}$ tending to $C_{0}$ must approach the divisor $E$ (see Figure 16(b)), but then $C_{0}$ must contain double fibers passing through the six nodes in $\mathbf{p}$, which is a contradiction. Suppose that $C_{0}$ contains a simple component $E$ and a double fiber $F^{\prime}$. Then the complementary trigonal curve $C_{0}^{\prime} \in|3 H|$ does not contain $E$ and has at least 6 singularities, among them at least 5 (namely, those in $\mathbf{p} \backslash F^{\prime}$ ) in general position in contradiction to Lemma 4.4(3). The case of a reduced, reducible $C_{0}$ is excluded by Lemma 5.2, since $C_{0}$ may have only $A D E$ singularities. Indeed, the total $\delta$-invariant of singular points in $\mathcal{F}_{2} \backslash E$ of a reduced curve from $|4 H|$ is at most 12 . Since $C_{0}$ has at least 8 singularities in $\mathcal{F}_{2} \backslash E$, the $\delta$-invariant of each of them is at most 5 , hence they all are simple. A singular point on $E$ can appear only when $C_{0}=C_{0}^{\prime} \cup E$. Since $C_{0}^{\prime} \cdot E=2$, we 
conclude that such a singular point is of type $A$ or $D$.

Next we set $\mathbf{p}=\operatorname{Sing}\left(C_{0}\right) \backslash d_{1}^{\prime \prime}\left(C_{0}\right), \mathbf{q}=\operatorname{Sing}\left(C_{0}\right) \cap d_{1}^{\prime \prime}\left(C_{0}\right)$, and introduce the stratum $S_{\mathbf{p}}\left(A_{3}\right) \subset \overline{\mathcal{R}\left(\mathcal{A}_{9}(6,0,2)\right)}$ of real rational curves with 7 nodes, one tacnode $A_{3}$, and almost fiberwise isotopic to $C_{0}$. Introduce the partial order on $S_{\mathbf{p}}\left(A_{3}\right)$ by setting $C^{\prime} \prec C^{\prime \prime}$ if $d_{0}\left(C^{\prime}\right), d_{1}^{\prime}\left(C^{\prime}\right), \ldots, d_{6}^{\prime}\left(C^{\prime}\right)$ are proper subsets of $d_{0}\left(C^{\prime \prime}\right), d_{1}^{\prime}\left(C^{\prime \prime}\right), \ldots, d_{6}^{\prime}\left(C^{\prime \prime}\right)$, respectively, and $d_{1}^{\prime \prime}\left(C^{\prime \prime}\right)$ is a proper subset of $d_{1}^{\prime \prime}\left(C^{\prime}\right)$. Consider the germ of the family $M_{\mathbf{p}, \mathbf{q}}\left(C_{0}\right) \subset S_{\mathbf{p}}\left(A_{3}\right)$. Lemmas $4.1(1,2)$ and 4.2 yield that $M_{\mathbf{p}, \mathbf{q}}\left(C_{0}\right)$ is smooth and one-dimensional, and that any curve $C^{\prime} \in$ $M_{\mathbf{p}, \mathbf{q}}\left(C_{0}\right) \backslash\left\{C_{0}\right\}$ intersects $C_{0}$ with multiplicity 4 at each node $z \in \mathbf{p}$, intersects $C_{0}$ in a neighborhood of the node in $\mathbf{q}$ at two points and in a neighborhood of the tacnode $q \in \mathbf{q}$ at 6 points. This, in particular, gives conditions (13) and (14) in Lemma 4.1(3). Hence, we obtain congruences (15) and inequality (16), provided that we verify the assumptions $\alpha_{0} \neq 0$ and $\beta_{0}^{2} \neq 4 \alpha_{0}^{2}$. Denote by $\widetilde{T}_{C_{0}} M_{\mathbf{p}, \mathbf{q}}\left(C_{0}\right)$ the tangent line to $M_{\mathbf{p}, \mathbf{q}}\left(C_{0}\right)$ in the linear system $|4 H|$. In the local coordinates $(x, y)$ in a neighborhood of the point $q$ chosen as in Lemma 4.1(3), we have $C_{0}=\{f=0\}$, $f=y^{2}-x^{4}$, and $C^{*}=\left\{f+\lambda f^{*}=0\right\}$ for $C^{*} \in \widetilde{T}_{C_{0}} M_{\mathbf{p}, \mathbf{q}}\left(C_{0}\right)$.

Now, if $\alpha_{0}=\beta_{0}=0$, then the monomials of $f^{*}$ under the Newton diagram of $f$ vanish, and we get $\left(C^{*} \cdot C_{0}\right)_{q} \geq 8$ contrary to (13). If $\alpha_{0}=0, \beta_{0} \neq 0$, we get that a generic curve $C^{*} \in \widetilde{T}_{C_{0}} M_{\mathbf{p}, \mathbf{q}}\left(C_{0}\right)$ has two local branches at $q: y=\xi x+O\left(x^{2}\right)$ and $y=\eta x^{3}+O\left(x^{4}\right)$. The first one passes outside the domain $-x^{2} \leq y \leq x^{2}$, and the second one within (see Figure 16(d), where $C^{*}$ is depicted by the dashed line). Notice that $C^{*}$ intersects $C_{0}$ only in $\operatorname{Sing}\left(C_{0}\right)$ : with multiplicity 4 at each node in $\mathbf{p}$, with multiplicity 2 and 6 at the node and tacnode on $\partial d_{1}^{\prime \prime}$, respectively. It follows that both the lower half-branch of $y=\xi x+O\left(x^{2}\right)$ and the left half-branch of $y=\eta x^{3}+O\left(x^{4}\right)$ extend so that both must pass through the node $z$ of $C_{0}$ on $\partial d_{1}^{\prime \prime}$ that contradicts the aforementioned equality $\left(C_{0} \cdot C^{*}\right)_{z}=2$.

At last, if $\alpha_{0} \beta_{0} \neq 0, \beta_{0}= \pm 2 \alpha_{0}$, then $f^{*}(x, y)= \pm 4 \alpha_{0} x\left(y-x^{2}+O\left(x^{3}\right)\right)$ and thus $\left.f^{*}\right|_{Q_{1}}=f^{*}\left(x, x^{2}\right)=x^{k}(\gamma+O(x)), \gamma \neq 0, k \geq 4$, which yields $\left(Q_{1}^{\prime} \cdot Q_{1}\right)_{U} \geq k-2 \geq 2$ contrary to the equality in (14). Hence, it follows that there exists a connected component $M$ of $M_{\mathbf{p}, \mathbf{q}}\left(C_{0}\right) \backslash\left\{C_{0}\right\}$ such that the real part of curves $C^{\prime} \in M$ looks as shown by the dashed line in Figure 16(c), in particular, $C_{0} \prec C^{\prime}$.

Now, having congruences (15) and inequality (16), as in the preceding case we obtain a limit curve $C_{1} \in \overline{S_{\mathbf{p}}\left(A_{3}\right)} \backslash S_{\mathbf{p}}\left(A_{3}\right)$, and moreover, we claim that it is irreducible and is attained as the disk $d_{1}^{\prime \prime}$ shrinks to a point. The proof of the irreducibility of $C_{1}$ literally coincides with that for $C_{0}$, presented above, except for the last step: the prohibition of a reduced, reducible $C_{1}$ not containing $E$ as component. On the last step we argue as follows. The total $\delta$-invariant of singular points in $\mathcal{F}_{2} \backslash E$ of a reduced curve from $|4 H|$ disjoint from $E$ does not exceed 12. Since $C_{1}$ has a least 7 singular points in $\mathcal{F}_{2} \backslash E$, their $\delta$-invariants do not exceed 6 . Furthermore a singularity of multiplicity 4 cannot appear, since there is no real fiber whose intersection points with a current curve $C^{\prime} \in S_{\mathbf{p}}\left(A_{3}\right), C^{\prime} \rightarrow C_{1}$, converge to one limit point (see Figure 16(c)). Then Lemmas 2.6 and 5.2 apply and prohibit such a possibility.

Thus, $C_{1}$ is irreducible, having 6 nodes, a singularity $A_{5}$, and the almost fiberwise type shown in Figure 15(c).

Step 2. Given $1 \leq s \leq 4$, denote by $S_{\mathbf{p}_{s}}\left(A_{5}, A_{2 s-1}\right) \subset \overline{\mathcal{R}\left(\mathcal{A}_{9}(6,0,2)\right)}$ the stratum formed by rational curves with singularities $A_{5}, A_{2 s-1}$ and $6-s$ nodes of the almost fiberwise isotopy type shown in Figure 17(a) and whose singular points outside the 


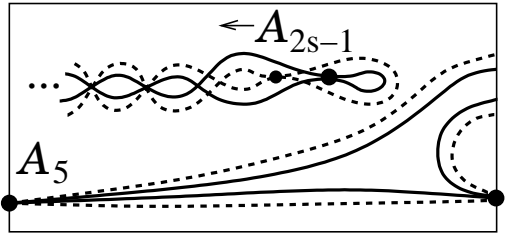

(a)

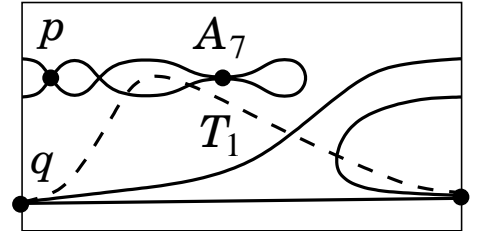

(b)

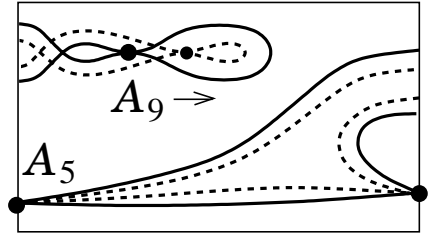

(c)

FiguRE 17

disk $d_{2}^{\prime}$ are in a fixed position $\mathbf{p}_{s}$. For example, the curve $C_{1}$ obtained in Step 1 belongs to $S_{\mathbf{p}_{1}}\left(A_{5}, A_{1}\right)$. By [26; Proposition 2.4(b)] we can suppose that the singularities $\mathbf{p}_{s}$ are in general position (including the two points infinitely near to singularity $\left.A_{5}\right)$. We introduce the partial order in $S_{\mathbf{p}_{s}}\left(A_{5}, A_{2 s-1}\right)$ by setting $C^{\prime} \prec C^{\prime \prime}$ if $d_{0}\left(C^{\prime}\right)$ and $d_{i}^{\prime}\left(C^{\prime}\right), 1 \leq i \leq 7-s, i \neq 2$, are proper subsets of $d_{0}\left(C^{\prime \prime}\right)$ and $d_{i}^{\prime}\left(C^{\prime \prime}\right), 1 \leq i \leq 7-s, i \neq 2$, respectively, and $d_{2}^{\prime}\left(C^{\prime \prime}\right)$ is a proper subset of $d_{2}^{\prime}\left(C^{\prime}\right)$. Using Lemma 4.2 as we did in Step 1, we derive that the family $M_{\mathbf{p}_{s}, \mathbf{q}}\left(C_{s}\right) \subset$ $S_{\mathbf{p}_{s}}\left(A_{5}, A_{2 s-1}\right)$ is smooth and one-dimensional, and then applying Lemma 4.1, we deduce that there exists a component $M$ of $M_{\mathbf{p}_{s}, \mathbf{q}}\left(C_{s}\right) \backslash\left\{C_{s}\right\}$ such that $C_{s} \prec C^{\prime} \in M$ (see $C^{\prime} \in M$ shown in Figure 17(a) by the dashed line).

As in Step 1, we obtain a curve $C_{s+1} \in \overline{S_{\mathbf{p}_{s}}\left(A_{5}, A_{2 s-1}\right)} \backslash S_{\mathbf{p}_{s}}\left(A_{5}, A_{2 s-1}\right)$, approximated by a sequence of curves from $\left\{C^{\prime} \in S_{\mathbf{p}_{s}}\left(A_{5}, A_{2 s-1}\right) \mid C_{s} \prec C^{\prime}\right\}$. We claim that $C_{s+1}$ is irreducible and is attained as the disk $d_{2}^{\prime}$ shrinks to a point. We prove this only for $s=4$, since for $s=1,2,3$ the same reasoning works well.

The set $\mathbf{p}_{4}$ consists of two points: of type $A_{1}$ and $A_{5}$ (for $C_{4}$ ) which we denote by $p$ and $q$ respectively (see Figure $17(\mathrm{~b})$ ). We denote a current curve approaching to $C_{5}$ along the family $S_{\mathbf{p}_{4}}\left(A_{5}, A_{7}\right)$ by $C^{\prime}$, and we set $d_{i}^{\prime}\left(C_{5}\right)=\lim _{C^{\prime} \rightarrow C_{5}} d_{i}^{\prime}\left(C^{\prime}\right)$, $i=1,2,3$. For a curve $D$ and a subset $\Omega \subset \mathcal{F}_{2}$, we define $\delta(D, \Omega)$ as the sum of $\delta$-invariants of those singular points of $D$ which belong to $\Omega$.

We list below all possibilities of a non-reduced, or reducible curve $C_{5}$ and bring this to contradiction.

Case 1. $C_{5}$ contains a multiple component $T_{1}$ belonging to $|H|,|H+F|$, or $|H+2 F|$. Then the intersection points of a current curve $C^{\prime}$ with a generic real fiber crossing the disc $d_{1}^{\prime}$ or $d_{3}^{\prime}$ move so that two points are approaching each other and the other two run away towards $E$ as $C^{\prime} \rightarrow C_{5}$ (see Figure 17(a)). Hence, $C_{5}$ contains $T_{1}$ with multiplicity 2 , and the real branch $\mathbb{R} T_{1}$ goes below the discs $d_{1}^{\prime}, d_{3}^{\prime}$. Since $T_{1}$ is smooth, and the lower arcs incident to $p$ are approaching $T_{1}$, we derive, first, that $p \notin T_{1}$ and, second, that these lower arcs, in the limit, doubly cover a segment of the fiber $F_{p}$, passing through $p$. Hence, $C_{5}$ splits off $2 F_{p}$, which particularly excludes the option of $T_{1} \in|H+2 F|$ and also yields that $E \subset C_{5}$.

Case 1.1. $C_{5}$ splits off $2 E$. Then the lower arcs of $C^{\prime}$ incident to $q$ are moving towards $E$ and also, in the limit, they doubly cover the fiber $F_{q}$ through $q$ (see Figure $17(\mathrm{a}))$. Hence, $C_{5}$ splits off $2 F_{q}$ that leaves the only possibility $T_{1} \in|H|$. In a neighborhood of $q$, choose local coordinates so that $C_{4}=\left\{y^{2}-x^{6}=0\right\}$. By construction, all curves in $S_{\mathbf{p}_{4}}\left(A_{5}, A_{7}\right)$ are locally given by $g=0$ with $g \in$ $\left\langle x^{6}, x^{3} y, y^{2}\right\rangle$ and the double fiber $2 F_{q}$ is given by $x^{2}=0$. Then $T_{1}$ is given by $h(x, y)=0$ such that $x^{2} h^{2} \in\left\langle x^{6}, x^{3} y, y^{2}\right\rangle$, which yields that $h \in\left\langle x^{2}, y\right\rangle$. Since the singular points on $\partial d_{2}^{\prime}$ must take the limit position on $T_{1}$, we derive that $T_{1}$ intersects the disc $d_{2}^{\prime}\left(C^{\prime}\right)$ for all $C^{\prime}$. This fact together with $T_{1} \cdot C^{\prime}=8$ yields 
that $T_{1}$ is quadratically tangent to the branches of $C_{4}$ at the point $q$ and the real part $\mathbb{R} T_{1}$ is located as shown in Figure $17(\mathrm{~b})$ by the dashed line. However, such a shape of $\mathbb{R} T_{1}$ is impossible: moving $T_{1}$ upward in the pencil of curves in $|H|$ that are quadratically tangent to the branches of $C_{4}$ at $q$, we monotonically extend the arc which is cut out of $\partial d_{2}^{\prime}$, and hence necessarily obtain more than $8=T_{1} \cdot C_{4}$ intersection points.

Case 1.2. $C_{5}$ splits off $E$ with multiplicity one (recall that $C_{5}$ contains also the double fiber $2 F_{p}$ ). Then $C_{5}$ splits off the double curve $2 T_{1}, T_{1} \in|H|$, and a curve $T_{1}^{\prime} \in|H|, T_{1}^{\prime} \neq T_{1}$. Since $T_{1}^{\prime}$ is smooth, $T_{1}$ must pass through $q$ and cross the disc $d_{2}^{\prime}$. Furthermore, $T_{1}$ must be tangent to $C_{4}$ at $q$. Indeed, otherwise, $T_{1}$ is locally given by $h=0$ with $h=x+\lambda y+O\left(x^{2}, y^{2}\right)$, and $T_{1}^{\prime}$ is given by $h^{\prime}=0$ with $h^{\prime}=y+O\left(x, y^{2}\right)$, but then $h^{\prime} h^{2}=x^{2} y+\ldots \notin\left\langle x^{6}, x^{3} y, y^{2}\right\rangle$. Then as above we decide that $\mathbb{R} T_{1}$ must be located as shown in Figure 17(b), which is impossible.

Case 2. $C_{5}$ contains the double component $E$ and no double components from $|H|,|H+F|$, or $|H+2 F|$. Then the lower arcs of $C^{\prime}$ incident to $q$ are moving towards $E$ and as above, in the limit, doubly cover the fiber $F_{q}$ through $q$ (see Figure 17(a)). Similarly, the upper arcs incident to $p$ approach $E$ and simultaneously doubly cover the fiber $F_{p}$ through $p$. Thus, $C_{5}$ splits off $2 E, 2 F_{p}$, and $2 F_{q}$. The remaining part $T_{2} \in|2 H|$ is reduced. Then we have $\delta\left(T_{2}, d_{2}^{\prime}\left(C_{5}\right)\right)=\delta\left(C_{5}, d_{2}^{\prime}\left(C_{5}\right)\right) \geq$ $\delta\left(C^{\prime}, d_{2}^{\prime}\left(C^{\prime}\right)\right)=\delta\left(A_{7}\right)+\delta\left(A_{1}\right)=5$ which is impossible for a reduced curve in $|2 H|$.

Case 3. $C_{5}$ contains $E$ with multiplicity one, a double fiber $2 F$, and a reduced curve $T_{3} \in|3 H|$ disjoint from $E$. If the double fiber $2 F$ intersects the disc $d_{2}^{\prime}\left(C_{5}\right)$, the the upper arcs of the discs $d_{1}^{\prime}$ and $d_{3}^{\prime}$ must converge to $E \cup F$ (see Figure $17(\mathrm{a}))$. Since the left endpoint $p \in \mathbf{p}_{4}$ of the upper arc of $d_{3}^{\prime}$ is fixed, this arc also approaches the fiber $F_{p}$, which means that $C_{5}$ contains the fiber $F_{p}$ as well contrary to the assumption made. Hence, $F$ does not intersect the disc $d_{2}^{\prime}$. Thus, $\delta\left(T_{3}, d_{2}^{\prime}\left(C_{5}\right)\right) \geq 5$ and in addition $T_{3}$ has either singularity $A_{5}$ at $q$, if $q \notin F$, or singularities at $p$ and $q$ if $p \notin F$, which finally yields $\delta\left(T_{3}\right) \geq 7$ which is impossible for a reduced curve $T_{3} \in|3 H|$ disjoint from $E$.

Case $4 . C_{5}$ is reduced, reducible.

Case 4.1. $C_{5}$ contains $E$ and a reduced curve $T_{3} \in|3 H+2 F|, T_{3} \not \supset E$. Note that $E T_{3}=2$. The situation when $T_{3}$ transversally intersects $E$ at two points is forbidden by Lemma 2.5. Otherwise, $C_{5}$ has at $E \cap T_{3}$ a simple singularity. A deformation of $C_{5}$ into $C^{\prime}$ induces a nodal deformation of that singular point. However, any nodal deformation of a simple singularity factors through a nodal equigeneric deformation (cf. the proof of Lemma 5.2), and hence the deformation $C_{5} \rightarrow C^{\prime}$ factors through a deformation $C_{5} \rightarrow E \cup T_{3}^{\prime}$, where $T_{3}^{\prime} \in|3 H+2 F|$ transversally intersects $E$ at two points, which was forbidden above.

Case 4.2. $C_{5}$ is reduced, $C_{5} \not \supset E$. We claim that $C_{5}$ must have only $A D E$ singularities or singularities of multiplicity 3 with $\delta \leq 7$, and hence a contradiction due to Lemmas 2.6 and 5.2.

Note that there is no real fiber such that all its intersection points with $C^{\prime}$ converge to one limit as $C^{\prime} \rightarrow C_{5}$. Hence $C_{5}$ cannot have singularities of multiplicity 4.

If $C_{5}$ splits into two or three components, then $\delta\left(C_{5}\right) \leq 11$, which together with relations $\delta\left(C_{5}, p\right) \geq 1, \delta\left(C_{5}, q\right) \geq 3$, and $\delta\left(C_{5}, d_{2}^{\prime}\left(C_{5}\right)\right) \geq 5$, yields that $\delta$-invariant of singularities of $C_{5}$ do not exceed 7 whence the required claim. 
If $C_{5}$ splits into four components $T_{1}^{(i)} \in|H|, i=1,2,3,4$, then in view of $T_{1}^{(i)} \cdot T_{1}^{(j)}=2$, the only non-simple singularity of multiplicity 3 , which may occur on $C_{5}$ is $J_{10}$.

Thus, $C_{5}$ is irreducible, and hence is as asserted in Lemma.

Lemma 5.4. There is no real rational algebraic curve $C \in|4 H|$ with singularities $A_{1}, A_{5}, A_{9}$ and the almost fiberwise isotopy type shown in Figure 15(b).

Proof. We argue on the contrary. Assume that there exists a curve $C$ as described in lemma. Using the Hilbert-Rohn-Gudkov method in the version of Section 4 (cf. the proof of Lemma 5.3), we will prove that this assumption leads to the existence one of the real rational curves of almost fiberwise isotopy types shown in Figure $14(\mathrm{a}, \mathrm{b})$ that are prohibited by Lemma 3.1. Let $\mathbf{p}$ be the $A_{5} \operatorname{singular}$ point of $C$, and $\mathbf{q}$ the remaining pair of singular points of $C$. In the notation of Step 2 in the proof of Lemma 5.3, $C$ belongs to the stratum $S_{\mathbf{p}}\left(A_{5}, A_{9}\right)$, in which we define the following partial order: $C^{\prime} \prec C^{\prime \prime}$ if $d_{0}\left(C^{\prime \prime}\right), d_{1}^{\prime}\left(C^{\prime \prime}\right)$, and $d_{2}^{\prime}\left(C^{\prime}\right)$ are proper subsets of $d_{0}\left(C^{\prime}\right)$, $d_{1}^{\prime}\left(C^{\prime}\right)$, and $d_{2}^{\prime}\left(C^{\prime \prime}\right)$, respectively. By Lemma 4.2 , the germ $M_{\mathbf{p}, \mathbf{q}}(C) \subset S_{\mathbf{p}}\left(A_{5}, A_{9}\right)$ is smooth and one-dimensional. Furthermore, Lemma 4.1 applies (for example, the verification of hypotheses of part (3) is similar to that performed in the proof of Lemma 5.3). From relations (14), (15), and (16), we derive that there exists a component $M$ of $M_{\mathbf{p}, \mathbf{q}}(C) \backslash\{C\}$ such that $C \prec C^{\prime}$ for all curves $C^{\prime} \in M$ (see Figure $17(\mathrm{c}))$.

As in the proof of Lemma 5.3 we obtain a curve $\hat{C} \in \bar{S}_{\mathbf{p}}\left(A_{5}, A_{9}\right) \backslash S_{\mathbf{p}}\left(A_{5}, A_{9}\right)$, approximated by a sequence of curves from $\left\{C^{\prime} \in S_{\mathbf{p}}\left(A_{5}, A_{9}\right) \mid C \prec C^{\prime}\right\}$. We claim that $\hat{C}$ is irreducible, and hence it takes one of the forms shown in Figure 14(a,b) when either the disk $d_{1}^{\prime}$ shrinks to a point, or the nodal singular point arrives to the fiber $F_{0}$.

First, we note that $\hat{C}$ cannot contain $E$ as component. Indeed, consider real fibers $F^{\prime}$ which are close the fiber $F_{q}$, passing through the point $q$ of type $A_{5}$. Then the intersection points of $F^{\prime}$ with the current curve $C^{\prime} \in S_{\mathbf{p}}\left(A_{5}, A_{9}\right), C^{\prime} \rightarrow \hat{C}$, do not tend to $E$ (see Figure 17(c)).

Second, $\hat{C}$ cannot contain a multiple component. Indeed, the movement of the intersection points of $C^{\prime}$ with the fibers $F^{\prime}$ discussed above shows that the multiplicity of a multiple component must be 2 . Furthermore, one of the intersection points of $C^{\prime}$ with a fiber crossing $d_{3}^{\prime}\left(C^{\prime}\right)$ runs away towards $E$ and does not approach other three points; hence $\hat{C}$ may contain only one double component $2 T_{1}, T_{1} \in|H|$. If $T_{1}$ does not pass through $q$ (singular point of type $A_{5}$ ), then the remaining part $T_{2}$ of $\hat{C}$, which is a reduced curve from $|2 H|$, must have singularity at least $A_{5}$ at $q$, what is impossible. Hence, $T_{1}$ passes through $q$. Moreover, the movement of the intersection points of $C^{\prime}$ with the fibers $F^{\prime}$ shows that $T_{1}$ must intersect each local branch of $C^{\prime}$ at $q$ with multiplicity $\geq 3$ being placed between them, and hence, in view of $\left(T_{1} \cdot C^{\prime}\right)_{q} \geq 6$ and $T_{1} C^{\prime}=8$, the curve $T_{1}$ cannot intersect any of the discs $d_{i}^{\prime}\left(C^{\prime}\right), i=1,2$. Hence, the singular point of $\hat{C}$, which is the limit of the singularity $A_{9}$ of $C^{\prime}$, is a singular point of $T_{2}$, what is impossible, since a reduced curve from $|2 H|$ cannot have singularity with $\delta$-invariant $\geq \delta\left(A_{9}\right)=5$.

Finally, we have to exclude the case of a reduced, reducible curve $\hat{C}$ having components from $|k H|, 1 \leq k \leq 3$, and this can be done in the same manner as we did in Case 4.2 in the proof of the irreducibility of the curve $C_{5}$ (end of the proof of Lemma 5.3). 
5.2. Prohibition of curves of types $\mathcal{A}^{\prime \prime}(4,0,3), \mathcal{A}^{\prime \prime}(3,1,3)$, and $\mathcal{A}^{\prime \prime}(3,0,3)$.

Suppose that there exists a smooth algebraic curve $C \in|4 H|$ of type $\mathcal{A}^{\prime \prime}(4,0,3)$, $\mathcal{A}^{\prime \prime}(3,1,3)$, or $\mathcal{A}^{\prime \prime}(3,0,3)$. Denote the arcs of the real branch of type $\mathcal{A}$ by $\rho_{1}, \rho_{2}, \rho_{3}, \rho_{4}$, numbering them from the top left to the bottom right in Figure 1. Let $\tau_{a}, \tau_{b}, \tau_{c}$

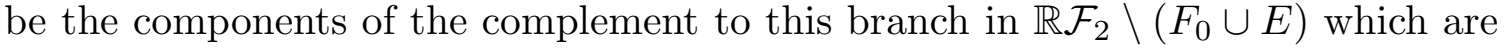
labeled by the respective letters $a, b, c$ in Figure 1(right). Denote the disks bounded by the ovals in the domains $\tau_{a}, \tau_{c}$ by $O_{i}^{a}, O_{j}^{c}$ respectively, numbering them from the middle to the sides (i.e., towards $F_{0}$ ).

Since $\operatorname{dim}|2 H|=8$, through any 8 points of $\mathcal{F}_{2} \backslash E$ one can draw a curve, belonging to $|2 H|$. Using this observation, we can degenerate $C$ into a curve with $\geq 8$ nodes as follows. Put $C_{0}=C$. Given a degeneration $C_{k}=\left\{f_{k}=0\right\}$ of $C$ with $0 \leq k \leq 7$ nodes, we consider the pencil $\left\{f_{k}+t g_{k}^{2}=0\right\}, t \geq 0$, where $\left\{g_{k}=0\right\} \in|2 H|$ passes through the nodes of $C_{k}$, and the sign is chosen so that the domain $\tau_{b}$ and the disks $O_{i}^{a}, O_{j}^{c}$ grow, and the disk bounded by an oval in the domain $\tau_{b}$ contracts when $t$ grows in $[0, \infty[$. Observe that, in this deformation, the intersection points with $F_{0}$ remain real until we encounter the first degeneration $C_{k+1}$ (which necessarily occurs due to the strongly monotone changes of the real geometry of the curve).

Let us analyze the geometry of $C_{k+1}$. Suppose that $k \leq 6$ and $C_{k}$ is irreducible with $k$ real nodes as its only singularities. By [25; Lemma 3.4] we can slightly deform $C_{k}$ bringing the nodes into a general position and making $C_{k}$ general in the linear subsystem of $|4 H|$ of curves having $k$ fixed nodes. Then by [25; Lemmas 3.5 and 3.6], $C_{k+1}$ is either an irreducible real curve with $k+1$ real nodes or splits into $E \cup C^{\prime}$, where $C^{\prime} \in|4 H-E|$ is an irreducible curve transversally crossing $E$ in two points. We, however, can exclude the latter option by choosing the auxiliary curve $\left\{g_{k}=0\right\} \in|2 H|$ passing through the $k \leq 6$ nodes and the two intersection point $C_{k} \cap F_{0}$, which are close to $E$. Suppose now that $k=7$, the curve $C_{7}$ is irreducible with 7 real nodes in general position, and $C_{7}$ is generic among curves with 7 fixed nodes. Again by [25; Lemmas 3.5 and 3.6], $C_{8}$ is either an irreducible curve with 8 real nodes, or $C_{8}=E \cup C^{\prime}$, where $C^{\prime} \in|4 H-E|$ is an irreducible curve having 7 real nodes in general position and intersecting $E$ transversally in two points, or $C_{8}=C_{8}^{(1)} \cup C_{8}^{(2)}$, where $C_{8}^{(1)}, C_{8}^{(2)} \in|2 H|$ are irreducible real curves intersecting in 8 real points. The second option is forbidden by Lemma 2.5.

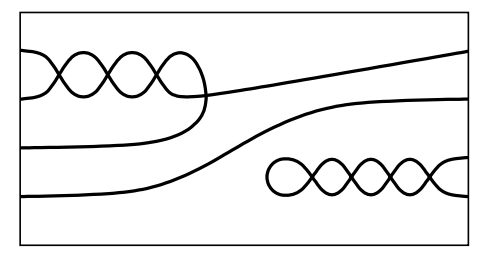

(a)

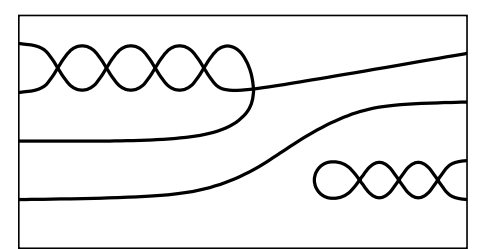

(b)

FIGURE 18

Suppose now that $C$ is of type $\mathcal{A}^{\prime \prime}(4,0,3)$, and $C_{8}$ is an 8-nodal curve constructed along the above algorithm. By Bézout bound to intersection with real fibers no two disks $O_{i}^{a}, O_{i+1}^{a}$ or the disk $O_{4}^{a}$ and the $\operatorname{arc} \rho_{1}$ (resp. two disks $O_{i}^{c}, O_{i+1}^{c}$ or the disk $O_{3}^{c}$ and the arc $\rho_{4}$ ) can join via two nodes. Furthermore, no isolated node can pop up in the domains $\tau_{a}$ and $\tau_{c}$, and no loop on arcs $\rho_{2}, \rho_{3}$ can appear, since 
an appropriate smoothing of nodes (see Lemma 4.3) would lead to an $(M-1)$ curve of type $\mathcal{A}^{\prime \prime}(5,0,3), \mathcal{A}^{\prime \prime}(4,0,4)$, or $\mathcal{A}^{\prime \prime}(4,1,3)$ forbidden in $[25,26]$. Hence, by Lemmas 2.3 and 2.4, the only possible shape of the curve $C_{8}$ constructed from $C$ is depicted in Figure 18(a,b). This curve $C_{8}$ does not admit a splitting $C_{8}^{(1)} \cup C_{8}^{(2)}$ with $C_{8}^{(1)}, C_{8}^{(2)} \in|2 H|$, since it has a global real branch intersecting $F_{0}$ in three points. Thus, $C_{8}$ is an irreducible elliptic curve. Consider the pencil $\left\{f_{8}+t g_{8}^{2}=0\right\}$ with $\left\{g_{8}=0\right\} \in|2 H|$ passing through the nodes of $C_{8}$ and the signs chosen so that the disks $O_{i}^{a}, O_{j}^{c}$ grow as $t$ grows in $[0, \infty[$. It follows that we arrive to a contradiction, since the geometry of the current curve changes in a strongly monotone way, and, on the other side, no degeneration $C_{9}$ is possible:

(i) A curve $C_{9}$ containing the double curve $E$ cannot occur, since the presence of 8 nodes in $\mathcal{F}_{2} \backslash E$, among which seven are in general position, leaves the only possibility $C_{9}=2 E \cup 2 C^{\prime}, C^{\prime} \in|H+2 F|$. However then the pencil $f_{8}+t g^{2}$ will consists of only reducible curves (indeed, $C_{9}=\left\{h^{2}=0\right\}$ implies $f_{8}+t g_{8}^{2}=\left(a g_{8}\right)^{2}-(b h)^{2}=\left(a g_{8}+b h\right)\left(a g_{8}-b h\right)$ for some $\left.a, b \in \mathbb{C}\right)$ contrary to the irreducibility of $C_{8}$.

(ii) A curve $C_{9}=E \cup C^{\prime}$, where $C^{\prime} \in|4 H-E|$ a reduced curve not containing $E$, is forbidden by Lemma 2.5. A curve $C_{9}=E \cup C^{\prime}$ with a non-reduced curve $C^{\prime} \in|4 H-E|$ not containing $E$ is not possible either. Indeed, then one would have $C^{\prime}=C_{1}^{\prime} \cup 2 C_{2}^{\prime}$, where either $C_{1}^{\prime} \in|H|, C_{2}^{\prime}|\in| H+F \mid$, or $C_{1}^{\prime} \in|H+2 F|, C_{2}^{\prime} \in|H|$. However, in the former case, $C_{1}^{\prime}$ is smooth, and $C_{2}^{\prime}$ passes through at most 6 nodes of $C_{8}$ (by Bézout), and in the latter case, $C_{2}^{\prime}$ passes through at most 4 nodes of $C_{8}$ (again by Bézout), while $C_{1}^{\prime}$ may have at most two singular points. It then follows that $C_{9}$ cannot contain $E$, and hence it cannot contain any fiber either. Thus, all components of $C_{9}$ belong to $|k H|, k=1,2,3,4$.

(iii) An irreducible curve $C_{9}$ with $\geq 9$ nodes is forbidden by Lemmas 2.3 and 2.4 .

(iv) A curve $C_{9}=C^{\prime} \cup C^{\prime \prime}$, where $C^{\prime}, C^{\prime \prime} \in|2 H|$, cannot occur. Indeed, if $C^{\prime}$ passes through all 8 nodes of $C_{8}$, then by Bézout so does $C^{\prime \prime}$, and hence all curves in the pencil $f_{8}+t g_{8}^{2}$ lie in the linear system spanned by $F_{1}^{2}, F_{1} F_{2}$, and $F_{2}^{2}$, where $F_{1}, F_{2}$ span the pencil in $|2 H|$ of curves passing through $\operatorname{Sing}(C)$, but this contradicts the irreducibility of $C_{8}$. If $C^{\prime}$ has a singularity at some node of $C_{8}$, then by Bézout it passes through at most 4 additional nodes of $C_{8}$, and hence $C^{\prime \prime}$ must be singular at 3 nodes of $C_{8}$ and either pass through two more nodes of $C_{8}$, or be singular in one more node of $C_{8}$, what is not possible, since all but one nodes of $C_{8}$ are in general position.

(v) At last, a curve $C_{9}$ cannot split as $C^{\prime} \cup C^{\prime \prime}$, where $C^{\prime} \in|H|, C^{\prime \prime} \in|3 H|$. Indeed, then $C^{\prime \prime}$ must have singularities at 4 nodes of $C_{8}$ and pass through the other 4 nodes of $C_{8}$. If $C_{8}^{\prime}, C_{8}^{\prime \prime}$ generate the pencil of curves in $|2 H|$ passing through $\operatorname{Sing}(C)$, then by Noether's $(A F+B G)$-theorem, $C^{\prime \prime}$ belong to the pencil spanned by $C_{8}^{\prime} G_{1}, C_{8}^{\prime \prime} G_{2}, G_{1}, G_{2} \in|H|$. It then follows that $C_{8}$ belongs to the linear subsystem in $|4 H|$ spanned by $F_{1}^{2}, F_{1} F_{2}$, and $F_{2}^{2}$ as in the preceding case in contradiction to the irreducibility of $C_{8}$.

Suppose that $C$ is of type $\mathcal{A}^{\prime \prime}(3,0,3)$ or $\mathcal{A}^{\prime \prime}(3,1,3)$. We similarly construct an 8-nodal curve $C_{8}$. Notice, first, that the Bézout type restriction mentioned in the preceding paragraph equally applies, and no isolated node can pop up in the domains $\tau_{a}$ and $\tau_{c}$, since otherwise we would get (via smoothing all nodes) 
either a curve of type $\mathcal{A}^{\prime \prime}(4,0,3)$ prohibited above. However, if we encounter a loop on arcs $\rho_{2}, \rho_{3}$ in the domain $\tau_{b}$, say, as in Figure 19(a), we continue to move along the corresponding pencil, turning the loop into an oval inside the domain $\tau_{b}$. Furthermore, if this oval apparently contracts to an isolated node, we also continue to move along the pencil (so that the node disappears). Thus, by Lemmas 2.3, 2.4, and 2.5, we arrive to a curve $C_{8}$ with the shape shown in Figure 8(a), maybe, with an oval in the domain $\tau_{b}$. By Lemma 2.1, this curve is reducible. Denote its components by $C_{1}$ and $C_{2}$ as in Lemma 2.1 .

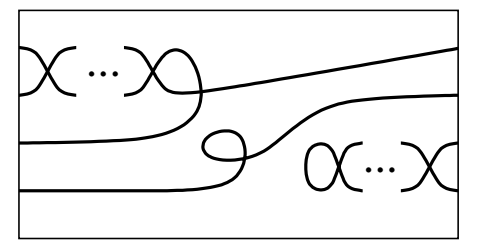

(a)

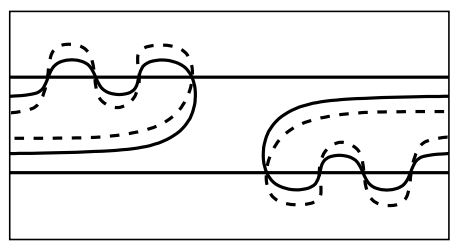

(b)

Figure 19

Consider the pencil $C_{8}+t C_{2}^{2}=C_{1}^{(t)} C_{2}$, oriented so that the domain $\tau_{b}$ and the disks $O_{i}^{a}, O_{j}^{c}$ grow as $t$ increases in [0, $\infty$ [ (see Figure 19(b), where the moving component $C_{1}^{(t)}, t>0$, is represented by the dashed line). The same arguments as above show that further degeneration is impossible which completes the proof of Theorem 1(a).

\section{Smoothings of Singularity $X_{21}$ Vs. CuRves on $\mathcal{F}_{2}$}

A plane curve singularity $X_{21}$ is a center of four smooth branches quadratically tangent to each other. Up to a local (equivariant in the real case) diffeomorphism, such a singular point is given by a function $f(x, y)=\sum_{i+2 j \geq 8} a_{i j} x^{i} y^{j}$, analytic in a neighborhood of the origin, where the lower $(1,2)$-homogeneous form $f_{8}(x, y)=$ $\sum_{i+2 j=8} a_{i j} x^{i} y^{j}$ is non-degenerate (i.e., has singularity only at the origin). Notice that $a_{04} \neq 0$, and the polynomial $\widehat{f}_{8}(x):=f_{8}(x, 1)$ has no multiple roots.

Suppose that $f$ (and $f_{8}$ ) is real. Put $B_{\delta}=D_{\delta, x} \times D_{\delta, y} \subset \mathbb{C}^{2}$, where $D_{\delta, x}$ and $D_{\delta, y}$ are closed disks of radius $\delta>0$, centered at the origin and located in the axes $x$ and $y$ respectively. We can define an equivariant surjection $\Phi_{\delta}: B_{\delta} \rightarrow \mathcal{F}_{2}$, which commutes with the rulings $\pi: \mathcal{F}_{2} \rightarrow E$ and $\pi_{\delta}: B_{\delta} \rightarrow D_{\delta, x}$, takes Int $B_{\delta}$ diffeomorphically onto $\mathcal{F}_{2} \backslash\left(F_{0} \cup E\right)$, takes each disk $\{\tau\} \times D_{\delta, y}$ onto a fiber $F \subset \mathcal{F}_{2}$ contracting $\{\tau\} \times \partial D_{\delta, y}$ to the point $F \cap E$, and takes the solid torus $\partial D_{\delta, x} \times D_{\delta, y}$ onto $F_{0}$ by contracting each circle $\partial D_{\delta, x} \times\{x\}, z \in D_{\delta, y}$, to a point and contracting $\partial D_{\delta, x} \times \partial D_{\delta, y}$ to the point $F_{0} \cap E$.

Choose $\delta$ so that $\{f=0\} \cap\left(D_{\delta, x} \times \partial D_{\delta, y}\right)=\varnothing$, and each open fiber of the ruling $\pi_{\delta}: B_{\delta} \rightarrow D_{\delta, x}$ intersects each branch of the curve $\{f=0\}$ at one point (we call $B_{\delta}$ a regular neighborhood of the singularity $f=0$ ). By a smoothing of a singularity $f=0$ we understand a one-parameter real analytic family $\psi_{t}=0$, $t \in[0, \varepsilon], \varepsilon>0$, such that $\psi_{0}=f$ and for $t \neq 0$ the analytic sets $\psi_{t}=0$ are smooth, disjoint from $D_{\delta, x} \times \partial D_{\delta, y}$, and intersecting each open fiber $\{\tau\} \times \operatorname{Int} D_{\delta, y}, \tau \in D_{\delta, x}$, with multiplicity 4 .

Two smoothings $\varphi_{t}^{(1)}, t \in[0, \varepsilon]$, and $\varphi_{t}^{(2)}, t \in[0, \varepsilon]$, of the singularity $f=0$ are called equivariant almost fiberwise isotopy (EAFI) equivalent, if $\left\{\varphi_{\varepsilon}^{(1)}=0\right\} \cap B_{\delta}$ 
can be connected with $\left\{\varphi_{\varepsilon}^{(2)}=0\right\} \cap B_{\delta}$ by an equivariant $C^{\infty}$ isotopy of $B_{\delta}$, which keeps $D_{\delta, x} \times \partial D_{\delta, y}$ and $\partial D_{\delta, x} \times D_{\delta, y}$ invariant and is such that the intersection with each fiber of the ruling $\pi_{\delta}: B_{\delta} \rightarrow D_{\delta, x}$ consists of 4 points (counting multiplicities).

Theorem 5. Given a real curve singularity $f=0$ of type $X_{21}$ and a regular neighborhood $B_{\delta}$, for any its smoothing $\psi_{t}=0, t \in[0, \varepsilon]$, there exist a smooth real algebraic curve $C \in|4 H|$ and an equivariant $C^{\infty}$ automorphism of $\mathcal{F}_{2}$, which commutes with $\pi: \mathcal{F}_{2} \rightarrow E$ and preserves $F_{0}$ and $E$, and such that $C$ is almost fiberwise isotopic to $\Phi_{\delta}\left(\left\{\psi_{\varepsilon}=0\right\} \cap B_{\delta}\right)$.

Remark 6.1. A similar result has been obtained for ordinary multiple singular points of order $\leq 5[29]$ and of order 6 [17]. We think that, in general, such a statement is not true.

Proof. (1) The strategy of the proof is as follows.

The Viro patchworking construction [31; Section 1.7] (see also [32]) associates with a real smooth curve $C \in|4 H|$ on $\mathcal{F}_{2}$ a smoothing of some real singular point of type $X_{21}$ related to the original curve $C$ as asserted in Theorem.

So, we need to prove the existence of a real smooth curve $C \in|4 H|$, which via patchworking gives a smoothing which is EAFI-equivalent to the given one. To this end, we invert the patchworking construction. Namely, given a smoothing $\psi_{t}=0, t \in[0, \varepsilon]$, of the considered singular point, we, first, obtain a singular curve $C^{\prime} \in|4 H|$, whose singularities can be locally smoothed out so that the resulting $C^{\infty}$ curve is almost fiberwise isotopy equivalent to $\Phi_{\delta}\left(\left\{\psi_{\varepsilon}=0\right\} \cap B_{\delta}\right)$, and, second, prove that all possible local singularity smoothings are independently realizable by variation of $C^{\prime}$ in the linear system $|4 H|$.

(2) Observe that there exists $\alpha \in \mathbb{R}$ such that after the coordinate change $(x, y) \mapsto\left(x, y+\alpha x^{2}\right)$ the coefficients of $f_{8}(x, y)$ satisfy the relation

$$
32 a_{04} a_{80}-2 a_{23} a_{61} \neq 0 .
$$

Indeed, the above coordinate change turns the left-hand side in (29) into

$$
\left(16 a_{04} a_{42}-6 a_{23}^{2}\right) \alpha^{2}+\left(24 a_{04} a_{61}-4 a_{23} a_{42}\right) \alpha+\left(32 a_{04} a_{80}-2 a_{23} a_{61}\right),
$$

which vanishes identically only if $f_{8}$ is the fourth power of a binomial. Since the spoken coordinate change does not affect the statement of Theorem, we may assume that (29) holds.

(3) Let

$$
g_{t}(x, y)=\sum_{i, j \geq 0} b_{i j}(t) x^{i} y^{j}, \quad b_{i j}(0)= \begin{cases}a_{i j}, & i+2 j \geq 8 \\ 0, & i+2 j<8\end{cases}
$$

be an analytic smoothing family. There are analytic functions $\alpha(t), \beta(t), \gamma(t)$, vanishing at 0 and such that $g_{t}(x+\gamma(t), y+\alpha(t)+\beta(t) x)$ has zero coefficients of $y^{3}$, $x y^{3}$, and $x^{7}$. Indeed, the Jacobian at $t=0$ of the corresponding system of equations on $\alpha, \beta, \gamma$ equals $4 a_{04}\left(32 a_{04} a_{80}-2 a_{23} a_{61}\right)$ which is nonzero in view of $(29)$. So, we can assume that $b_{03}=b_{13}=b_{70} \equiv 0$.

(4) Fix some $c_{0}>0$ and define $\tau(t)>0$ so that

$$
\sum_{i+2 j<8}\left|b_{i j}(t)\right| \tau(t)^{8-i-2 j}=c_{0}, \quad t>0
$$


Since $\sum_{i+2 j<8}\left|b_{i j}(t)\right|>0$ and $b_{i j}(0)=0$ as long as $i+2 j<8$, the function $\tau(t)$ is well-defined and satisfies $\lim _{t \rightarrow 0} \tau(t)=\infty$. In the family

$$
\begin{gathered}
\tau(t)^{8} g_{t}\left(x \tau(t)^{-1}, y \tau(t)^{-2}\right)=\sum_{i+2 j>8} b_{i j}(t) \tau(t)^{-(i+2 j-8)} x^{i} y^{j} \\
+\sum_{i+2 j=8} b_{i j}(t) x^{i} y^{j}+\sum_{i+2 j<8} b_{i j}(t) \tau(t)^{8-i-2 j} x^{i} y^{j},
\end{gathered}
$$

the first sum converges to zero and the second sum converges to $f_{8}(x, y)$ as $t \rightarrow 0$. Moreover, in view of (30), there exists a sequence $t_{n} \rightarrow 0, n=1,2, \ldots$, such that the third sum converges as well so that

$$
\lim _{n \rightarrow \infty} \tau\left(t_{n}\right)^{8} g_{t_{n}}\left(x \tau\left(t_{n}\right)^{-1}, y \tau\left(t_{n}\right)^{-2}\right)=f_{8}(x, y)+\sum_{i+2 j<8} a_{i j} x^{i} y^{j}=: G(x, y)
$$

where

$$
\sum_{i+2 j<8}\left|a_{i j}\right|=c_{0}>0 \quad \text { and } \quad a_{03}=a_{13}=a_{70}=0 .
$$

(5) If $G(x, y)=0$ is non-singular in $\mathbb{C}^{2}$, then it defines in $\mathcal{F}_{2}$ a smooth real curve belonging to the linear system $|4 H|$ and EAFI equivalent to $\Phi\left(\left\{g_{t}(x, y)=0\right\}\right)$ for $t \neq 0$. Suppose that $G(x, y)=0$ is singular in $\mathbb{C}^{2}$. Then

(i) $G(x, y)=0$ has no singularity of type $X_{21}$ in $\mathbb{C}^{2}$ in view of (31).

(ii) By construction, there is a (small) real deformation of $G(x, y)$, which smooths out all singular points of $G=0$ in $\mathbb{C}^{2}$ and, up to coordinate change, coincides with the given smoothing family.

Denote by $\mathcal{P}(k) \subset \mathbb{C}[x, y]$ the linear subspace spanned by the monomials $x^{i} y^{j}$ with $i+2 j=k$, and put

$$
\mathcal{P}_{(k)}=\bigoplus_{i \leq k} \mathcal{P}(i), \quad \mathcal{P}^{(k)}=\bigoplus_{i \geq k} \mathcal{P}(i)
$$

It follows from Lemma 6.2 below that $\mathcal{P}_{(8)}$ induces a joint versal deformation of all the singular points of $G=0$ in $\mathbb{C}^{2}$, i.e., by a small variation of coefficients of the monomials $x^{i} y^{j}, i+2 j \leq 8$, one can independently realize prescribed (up to local diffeomorphism) smoothing families for all singular points of $G=0$ in $\mathbb{C}^{2}$. Thus, we can obtain a polynomial $\widetilde{G}(x, y)=\sum_{i+2 j \leq 8} \widetilde{a}_{i j} x^{i} y^{j}$ close to $G(x, y)$, which defines in $\mathcal{F}_{2}$ a real curve EAFI equivalent to $\Phi\left(\left\{g_{t}(x, y)=0\right\}\right), t \neq 0$.

Lemma 6.2. Let $G=0$ where

$$
G(x, y)=f_{8}(x, y)+\sum_{i+2 j<8} a_{i j} x^{i} y^{j} \quad \text { and } f_{8}(x, y)=\sum_{i+2 j=8} a_{i j} x^{i} y^{j}
$$

be a reduced curve in $\mathbb{C}^{2}$ that has no singularity $X_{21}$, and the quasi-homogeneous polynomial $f_{8}$ is non-degenerate (i.e. splits into the product of four distinct binomoials). Then the linear space $\mathcal{P}_{(8)} \subset \mathbb{C}[x, y]$ spanned by the monomials $x^{i} y^{j}$, $0 \leq i+2 j \leq 8$, induces a joint versal deformation of all singularities of the curve $G=0$. 
Proof. As shown in Part (2) of the proof of Theorem 5, we can suppose that the coefficients of $f_{8}$ satisfy relation (29). Then we proceed in several steps.

(1) It is well-known that a versal deformation of each singular point $\left(x_{0}, y_{0}\right) \in$ $\operatorname{Sing}(G=0)$ is generated by any basis of the quotient $\mathbb{C}\left\{x-x_{0}, y-y_{0}\right\} /\left\langle G, G_{x}, G_{y}\right\rangle$, where $\mathbb{C}\{*, *\}$ denotes the ring of convergent power series, and $\left\langle G, G_{x}, G_{y}\right\rangle$ is the ideal generated by $G, G_{x}, G_{y}$ (Tjurina ideal). Thus, it is enough to prove the surjectivity of the following two projections:

$$
\begin{gathered}
\operatorname{pr}_{1}: \mathbb{C}[x, y] /\left\langle G, G_{x}, G_{y}\right\rangle \rightarrow \underset{\left(x_{0}, y_{0}\right) \in \operatorname{Sing}(G=0)}{\bigoplus} \mathbb{C}\left\{x-x_{0}, y-y_{0}\right\} /\left\langle G, G_{x}, G_{y}\right\rangle, \\
\operatorname{pr}_{2}: \mathcal{P}_{(8)} \rightarrow \mathbb{C}[x, y] /\left\langle G, G_{x}, G_{y}\right\rangle .
\end{gathered}
$$

(2) To prove the surjectivity of $\mathrm{pr}_{1}$, introduce the zero-dimensional subscheme $Z \subset \mathcal{F}_{2}$, concentrated at $\operatorname{Sing}(G=0)$ and defined by the local Tjurina ideal at each point. Then we have an exact sequence of sheaves

$$
0 \rightarrow \mathcal{J}_{Z / \mathcal{F}_{2}}(n D) \rightarrow \mathcal{O}_{\mathcal{F}_{2}}(n D) \rightarrow \mathcal{O}_{Z} \rightarrow 0
$$

$D$ any ample divisor. Since for $n \gg 0, H^{1}\left(\mathcal{J}_{Z / \mathcal{F}_{2}}(n D)\right)=0$, we get

$$
\begin{aligned}
& H^{0}\left(\mathcal{O}_{\mathcal{F}_{2}}(n D)\right) / H^{0}\left(\mathcal{J}_{Z / \mathcal{F}_{2}}(n D)\right) \simeq H^{0}\left(\mathcal{O}_{Z}\right) \\
& \quad=\bigoplus_{\left(x_{0}, y_{0}\right) \in \operatorname{Sing}(G=0)} \mathbb{C}\left\{x-x_{0}, y-y_{0}\right\} /\left\langle G, G_{x}, G_{y}\right\rangle
\end{aligned}
$$

which, in fact, yields that $\mathrm{pr}_{1}$ is an isomorphism.

(3) Now we prove the surjectivity of $\mathrm{pr}_{2}$.

Performing a real coordinate change $(x, y) \mapsto\left(x, y+\alpha x^{2}\right)$ (which does not affect the considered statement) we can set the coefficient $a_{23}$ of $x^{2} y^{3}$ to zero, while keeping $a_{03}=a_{13}=0$ and the properties (i), (ii) of step (5) of the proof of Theorem 5 .

Write $G=f_{8}+\sum_{k<8} f_{k}, f_{k} \in \mathcal{P}(k), k \geq 0$, and put $k_{0}=\max \left\{k<8 \mid f_{k} \neq 0\right\}$ (notice that $k_{0}$ is well-defined, since $G \neq f_{8}$ ).

Since $f_{8}$ is non-degenerate, the derivatives $f_{8, x}, f_{8, y}$ are coprime, which immediately yields that the ideal $\left\langle f_{8, x}, f_{8, y}\right\rangle \subset \mathbb{C}[x, y]$ contains the ideal $\mathcal{P}^{(11)}=$ $\left\langle x^{i} y^{j}, i+2 j \geq 11\right\rangle$, and hence any polynomial in $\mathcal{P}^{(11)}$ equals $A G_{x}+B G_{y}$ modulo $\mathcal{P}_{(10)}$ with appropriate $A, B \in \mathbb{C}[x, y]$. Thus, we complete the proof when showing that any polynomial in $\mathcal{P}(s), s=9,10$, can be represented as $A G+B G_{x}+C G_{y}$ modulo $\mathcal{P}_{(s-1)}$ with some $A, B, C \in \mathbb{C}[x, y]$.

(3i) Suppose that either $k_{0}<7$, or $k_{0}=7$ and $f_{7} \notin \operatorname{Span}_{\mathbb{C}}\left\{f_{8, x}, x f_{8, y}\right\}$. We claim that in such a case

$$
f_{k_{0}} \mathcal{P}\left(s-k_{0}\right)+f_{8, x} \mathcal{P}(s-7)+f_{8, y} \mathcal{P}(s-6)=\mathcal{P}(s) \quad \text { for } \quad s=9,10
$$

Indeed, a violation of one of the two relations (33) enforces

$$
f_{k_{0}} \in\left\langle f_{8, x}, f_{8, y}\right\rangle .
$$


We demonstrate this in the case of $k_{0}=7$ and $s=10$; the other cases are treated in the same manner. In view of $\operatorname{dim} \mathcal{P}(10) /\left(f_{8, x} \mathcal{P}(3)+f_{8, y} \mathcal{P}(4)\right)=1$, the considered situation reduces to equalities

$$
x y f_{7}=A_{3} f_{8, x}+A_{4} f_{8, y}, \quad x^{3} f_{7}=B_{3} f_{8, x}+B_{4} f_{8, y}, \quad A_{i}, B_{i} \in \mathcal{P}(i), i \geq 0 .
$$

Since $f_{8, x}, f_{8, y}$ are coprime, we get

$$
A_{3}=y A_{1}, B_{3}=x^{2} A_{1}, \quad A_{4}=x^{2} B_{2}, B_{4}=y B_{2},
$$

and hence $x f_{7}=\alpha x f_{8, x}+\left(\beta x^{2}+\gamma y\right) f_{8, y}$, where $\gamma=0$ due to $a_{04} \neq 0$, and $(34)$ follows.

Now we show that (34) cannot hold. For $k_{0}=7$, (34) would contradict the assumption $f_{7} \notin \operatorname{Span}_{\mathbb{C}}\left\{f_{8, x}, x f_{8, y}\right\}$ made in the beginning of step (3i). For $k_{0}=6$, (34) would yield $f_{6}=c f_{8, y}, c \neq 0$, contrary to the fact that $a_{04} \neq 0$ and the coefficient of $y^{3}$ in $f_{6}$ vanishes by construction. Finally, for $k_{0}<6,(34)$ is excluded for the degree reason.

Having (33) proven, we proceed as follows. Pick an arbitrary polynomial $P_{s} \in$ $\mathcal{P}(s), 9 \leq s \leq 10$, and represent it as $P_{s}=A_{s-k_{0}} f_{k_{0}}+B_{s-7} f_{8, x}+B_{s-6} f_{8, y}$. Then by Euler formula, the leading $(1,2)$-homogeneous form of the polynomial $8 G-x G_{x}-2 y G_{y}$ is $\left(8-k_{0}\right) f_{k_{0}}$, and hence

$$
P_{s}=\frac{8 A_{s-k_{0}}}{8-k_{0}} G+\left(B_{s-7}-\frac{x A_{s-k_{0}}}{8-k_{0}}\right) G_{x}+\left(B_{s-6}-\frac{2 y A_{s-k_{0}}}{8-k_{0}}\right) G_{y} \bmod \mathcal{P}_{(s-1)},
$$

and we are done.

(3ii) Suppose that $k_{0}=7$ and $f_{7} \in\left\langle f_{8, x}, f_{8, y}\right\rangle$. Since $a_{13}=a_{23}=3$, we have $f_{7}=\alpha f_{8, x}$ for some $\alpha \in \mathbb{C}$.

Assume now that there exists $0 \leq m \leq 6$ such that

$$
(8-k) f_{k}=\alpha f_{k+1, x} \text { for all } m<k<7, \quad \text { and } f_{m}^{\prime}=(8-m) f_{m}-\alpha f_{m+1, x} \neq 0 \text {. }
$$

The argument of Step (3i) shows that

$$
f_{m}^{\prime} \mathcal{P}(s-m)+f_{8, x} \mathcal{P}(s-7)+f_{8, y} \mathcal{P}(s-6)=\mathcal{P}(s), \quad s=9,10 .
$$

So, represent any polynomial $P_{s} \in \mathcal{P}(s)$ as $P_{s}=A_{s-m} f_{m}^{\prime}+B_{s-7} f_{8, x}+B_{s-6} f_{8, y}$, and notice that the leading $(1,2)$-homogeneous form of the polynomial $8 G-x G_{x}-$ $2 y G_{y}-\alpha G_{x}$ is $f_{m}^{\prime}$. Hence

$$
P_{s}=A_{s-m}\left(8 G-x G_{x}-2 y G_{y}-\alpha G_{x}\right)+B_{s-7} G_{x}+B_{s-6} G_{y} \quad \bmod \mathcal{P}_{(s-1)} .
$$

It remains to consider the case when $(8-k) f_{k}=\alpha f_{k+1, x}$ for all $k<7$. However, then

$$
G(x, y)=f_{8}(x, y)+\sum_{k<8} f_{k}(x, y)=f_{8}(x, y)+\sum_{i>0} \frac{\alpha^{i}}{i !} \frac{\partial^{i}}{\partial x^{i}} f_{8}(x, y)=f_{8}(x+\alpha, y)
$$

which contradicts the assumption that $G(x, y)=0$ has no singularity $X_{21}$ in $\mathbb{C}^{2}$. 


\section{REFERENCES}

1. A. Akyol, A. Degtyarev, Geography of irreducible plane sextics, Proc. London Math. Soc. (to appear).

2. V. I. Arnol'd, S. M. Gusein-Zade, A. N. Varchenko, Singularities of differentiable maps, vol. 1, Birkhäuser Verlag, Boston, Basel, Stuttgart, 1985.

3. E. Artal Bartolo, J. Carmona Ruber, J. I. Cogolludo Augustin, On sextic curves with big Milnor number, Trend in Singularities, Trends Math., Birkhäuser, Basel, 2002, pp. 1-29.

4. E. Brugallé, Symmetric plane curves of degree 7: Pseudoholomorphic and algebraic classifications, J. reine angew. Math. 612 (2007), 129-171.

5. L. Caporaso, J. Harris, Parameter spaces for curves on surfaces and enumeration of rational curves, Compositio Math. 113 (1998), no. 2, 155-208.

6. A. Degtyarev, On the Artal-Carmona-Cogolludo Construction, J. Knot Theory Ramifications 23:5 (2014), Article ID 1450028, 35 p.

7. I. V. Dolgachev, Classical algebraic geometry: a modern view, Cambridge Univ. Press, 2013.

8. S. Fiedler-Le Touzé, S. Yu. Orevkov, A flexible affine $M$-sextic non-realizable algebraically, J. Algebraic Geom. 11 (2002), no. 2, 293-310.

9. G.-M. Greuel, U. Karras, Families of varieties with prescribed singularities, Compos. Math. 69 (1989), 83-110.

10. G.-M. Greuel, C. Lossen, and E. Shustin, Introduction to singularities and deformations, Springer, 2007.

11. D.A. Gudkov, G.A. Utkin, and M.L. Tai, The complete classification of nondecomposing curves of the 4 th order, Mat. Sb. 69 (111) (1966,), 222-256.

12. D. A. Gudkov and E. I. Shustin, On the intersection of the close algebraic curves, Dold, A.; Eckmann, B. eds.: Topology (Leningrad, 1982), Lect. Notes Math., vol. 1060, Springer, 1984, pp. 278-289.

13. V. M. Kharlamov, S. Yu. Orevkov, E. I. Shustin, Singularity which has no M-smoothing, E. Bierstone (ed.) et al., The Arnoldfest. Proc. Conf. in honour of V. I. Arnold for his 60th birthday, Toronto, Canada, June 15-21, 1997., Fields Inst. Commun. 24, Amer. Math. Soc., Providence, RI, 1999, pp. 273-309.

14. V. M. Kharlamov, O. Ya. Viro, Extensions of the Gudkov-Rokhlin congruence, Topology and geometry, Rohlin Semin. 1984-1986, Lect. Notes Math. 1346,, Springer, 1988, pp. 687-717.

15. A. B. Korchagin, New M-curves of degrees 8 and 9, Sov. Math. Dokl. 39 (1989), no. 3, $569-572$.

16. A. B. Korchagin, Construction of new curves of 9th degree, Real Algebraic Geometry (Proc. Conf., Rennes/Fr. 1991), Lect. Notes Math. 1524,, Springer, 1992, pp. 296-307.

17. A. B. Korchagin, E. I. Shustin, Affine curves of degree 6 and smoothings of a non-degenerate sixth order singular point, Math. USSR, Izv. 33 (1989), 501-520.

18. E. I. Moody, Notes on the Bertini involution, Bull. Amer. Math. Soc. 49 (1943), 433-436.

19. S. Yu. Orevkov, Classification of flexible $M$-curves of degree 8 up to isotopy, GAFA - Geom. and Funct. Anal. 12 (2002), 723-755.

20. S. Yu. Orevkov, Construction of arrangements of an $M$-quartic and an $M$-cubic with a maximal intersection of an oval and the odd branch, Bulletin (vestnik) of Nizhni Novgorod State University, Ser. Math. Modeling and Optimal Control, issue 1(25) (2002), 12-48 (Russian); English transl., http://picard.ups-tlse.fr/ orevkov/j3o4e.pdf.

21. S. Yu. Orevkov, Riemann existence theorem and construction of real algebraic curves, Ann. Fac. Sci. de Toulouse. Math. (6), 12 (2003), 517-531.

22. S. Yu. Orevkov, Quasipositivity problem for 3-braids, Turkish Journal of Math. 28 (2004), 89-93.

23. S. Yu. Orevkov, Arrangements of an $M$-quintic with respect to a conic that maximally intersects its odd branch, St. Petersburg Math. J. 19:4 (2008), 625-674.

24. S.Yu. Orevkov, Algorithmic recognition of quasipositive braids of algebraic length two, J. of Algebra 423 (2015), 1080-1108.

25. S. Yu. Orevkov and E. I. Shustin, Flexible, algebraically unrealizable curves: Rehabilitation of the Hilbert-Rohn-Gudkov approach, J. reine angew. Math. 551 (2002), 145-172.

26. S. Yu. Orevkov and E. I. Shustin, Pseudoholomorphic, algebraically unrealizable curves, Moscow Math. J. 3 (2003), 1053-1083.

27. J.-J. Risler, Un analogue local du théorème de Harnack, Invent. Math. 89 (1987), 119-137. 
28. E. I. Shustin, The Hilbert-Rohn method and smoothings of real algebraic curve singular points, Sov. Math. Doklady 31 (1983), no. 2, 282-286.

29. E. I. Shustin, Versal deformations in the space of plane curves of fixed degree, Function. Anal. Appl. 21 (1987), 82-84.

30. E. Shustin, Gluing of singular and critical points, Topology 37 (1998), no. 1, 195-217.

31. O. Viro, Patchworking Real Algebraic Varieties, Preprint at arXiv:math.AG/0611382.

32. O. Ya. Viro, Real algebraic plane curves: constructions with controlled topology, Leningrad Math. J. 1 (1990), 1059-1134.

33. J.-Y. Welschinger, Courbes algébriques réelles et courbes flexibles sur les surfaces réglées de base $\mathbb{C} P^{1}$, Proc. London Math. Soc. (3) 85 (2002), 367-392.

Steklov Mathematical Institute, Gubkina 8, 119991, Moscow, Russia;

imt, Université Paul Sabatier, 118 route de Narbonne, 31062, Toulouse, France;

National Research University Higher School of Economics, Russian Federation, Vavilova 7, Moscow, 117312 Russia

E-mail address: orevkov@math.ups-tlse.fr

School of Mathematical Sciences, Tel Aviv University, Ramat Aviv, 69978 Tel Aviv, ISRAEL

E-mail address: shustin@math.tau.ac.il 